\title{
CORECURSIVE ALGEBRAS, CORECURSIVE MONADS AND BLOOM MONADS
}

\author{
JIŘÍ ADÁMEK ${ }^{a}$, MAHDIE HADDADI $^{b}$, AND STEFAN MILIUS $^{c}$ \\ ${ }^{a}$ Institut für Theoretische Informatik, Technische Universität Braunschweig, Germany \\ e-mail address: adamek@iti.cs.tu-bs.de \\ ${ }^{b}$ Department of Mathematics, Statistics and Computer Science, Semnan University, Semnan, Iran \\ e-mail address: mahdiehaddadi7@gmail.com \\ ${ }^{c}$ Lehrstuhl für Theoretische Informatik, Friedrich-Alexander-Universität Erlangen-Nürnberg, Ger- \\ many \\ e-mail address: mail@stefan-milius.eu
}

\begin{abstract}
An algebra is called corecursive if from every coalgebra a unique coalgebra-toalgebra homomorphism exists into it. We prove that free corecursive algebras are obtained as coproducts of the terminal coalgebra (considered as an algebra) and free algebras. The monad of free corecursive algebras is proved to be the free corecursive monad, where the concept of corecursive monad is a generalization of Elgot's iterative monads, analogous to corecursive algebras generalizing completely iterative algebras. We also characterize the Eilenberg-Moore algebras for the free corecursive monad and call them Bloom algebras.
\end{abstract}

\section{INTRODUCTION}

The study of structured recursive definitions is fundamental in many areas of computer science. This study can use algebraic methods extended by suitable recursion concepts. One such example are completely iterative algebras: these are algebras in which recursive equations with parameters have unique solutions, see [28]. In the present paper we study corecursive algebras. These are $H$-algebras for a given endofunctor $H$ in which recursive equations without parameters have unique solutions. Equivalently, for every coalgebra there exists a unique coalgebra-to-algebra morphism. The dual concept, recursive coalgebra, was introduced by G. Osius in [29], and for endofunctors weakly preserving pullbacks P. Taylor proved that this is equivalent to being parametrically recursive, see [32]. In the dual situation, since weak preservation of pushouts is rare, the concepts of corecursive algebra and completely iterative algebra usually do not coincide. The former was studied by V. Capretta, T. Uustalu and V. Vene [21], and various counter-examples demonstrating e.g. the difference of the two concepts for algebras can be found there. In the present paper we contribute to the development of the mathematical theory of corecursive algebras. The goal

2012 ACM CCS: [Theory of computation]: Semantics and reasoning-Program semanticsCategorical semantics.

Key words and phrases: corecursive algebra, corecursive monads, Bloom monads, iteration theory.

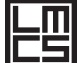
IN COMPUTER SCIENCE
DOI:10.2168/LMCS-10(3:19)2014
(C) J. Adámek, M. Haddadi, and S. Milius (c) Creative Commons 
is to eventually arrive at a useful body of results and constructions for these algebras. A major ingredient of any theory of algebraic structures is the study of how to freely endow an object with the structure of interest. So the main focus of the present paper are corecursive $H$-algebras freely generated by an object $Y$. Let $F Y$ denote the free $H$-algebra on $Y$ and let $T$ be the terminal $H$-coalgebra (which, due to Lambek's Lemma, can be regarded as an algebra). We prove that the coproduct of these two algebras

$$
M Y=T \oplus F Y
$$

is the free corecursive algebra on $Y$. Here $\oplus$ is the coproduct in the category of $H$-algebras. For example for the endofunctor $H X=X \times X$ the algebra $M Y$ consists of all (finite and infinite) binary trees with finitely many leaves labelled in $Y$.

We also introduce the concept of a corecursive monad. This is a weakening of completely iterative monads of C. Elgot, S. Bloom and R. Tindell [23] analogous to corecursive algebras as a weakening of completely iterative ones. The monad $Y \mapsto M Y$ of free corecursive algebras is proved to be corecursive, indeed, this is the free corecursive monad generated by $H$. For endofunctors of Set we also prove the converse: whenever $H$ generates a free corecursive monad, then it has free corecursive algebras (and the free monad is then given by the corresponding adjunction).

We characterize the Eilenberg-Moore algebras for the free corecursive monad: these are $H$-algebras in which every recursive equation without parameters has a solution (not necessarily unique), and which allow a functorial choice of solutions. We call these algebras Bloom algebras; they are analogous to the complete Elgot algebras of [9] where the corresponding monad was the free completely iterative monad on $H$.

We also study the finitary versions of our concepts. An algebra $A$ is called finitary corecursive if all coalgebras on finitely presentable objects have a unique coalgebra-to-algebra morphism in to $A$. And finitary corecursive monads are defined analogously. Every finitary endofunctor $H$ is proved to generate a free finitary corecursive monad $\mathbb{M}_{H}$. We form the free strict functor $H_{\perp}=H+1$ on $H$ and obtain a monad $\mathcal{M}^{*}$ on the category of finitary functors given by

$$
\mathcal{M}^{*}(H)=\mathbb{M}_{H_{\perp}}
$$

The Eilenberg-Moore algebras for $\mathcal{M}^{*}$ are called Bloom monads. They correspond to iteration theories of Bloom and Ésik: recall from [11] that the latter are precisely the EilenbergMoore algebras for the free-iteration-theory monad. Bloom monads are monads $\mathbb{S}$ equipped with an operation $\dagger$ assigning to every finitary non-parametric equation morphism a solution in free algebras for $\mathbb{S}$. This operation satisfies precisely the equational properties that non-parametric iteration in Domain Theory satisfies. We list some of those equational properties. The question whether our list is complete is open.

This paper is a revised and extended version of the conference paper [4]. Here we added all technical details and proofs, and our discussion of the equational properties of Bloom monads and their properties in new.

Acknowledgments. We are grateful to Zoltán Ésik for a substantial contribution to the discussion of equations in Bloom monads. And to Paul Levy who suggested that Proposition 3.4 holds. 


\section{Corecursive Algebras}

The following definition is the dual of the concept introduced by G. Osius in [29] and studied by P. Taylor [32, 31. We assume throughout the paper that a category $\mathcal{A}$ and an endofunctor $H: \mathcal{A} \rightarrow \mathcal{A}$ are given. We denote by $\operatorname{Alg} H$ the category of algebras $a: H A \rightarrow A$ and homomorphisms, and by Coalg $H$ the category of coalgebras $e: X \rightarrow H X$ and homomorphisms. A coalgebra-to-algebra morphism from the latter to the former is a morphism $f: X \rightarrow A$ such that $f=a \cdot H f \cdot e$.

Definition 2.1. An algebra $a: H A \rightarrow A$ is called corecursive if for every coalgebra $e$ : $X \rightarrow H X$ there exists a unique coalgebra-to-algebra homomorphism $e^{\dagger}: X \rightarrow A$. That is, the square

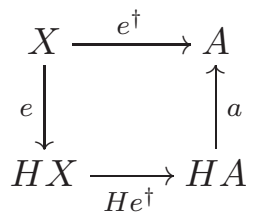

commutes. We call $e$ an equation morphism and $e^{\dagger}$ its solution.

Remark 2.2. For an endofunctor on Set, we can view $e: X \rightarrow H X$ as a system of recursive equations using variables from the set $X$, and $e^{\dagger}: X \rightarrow A$ is the solution of the system. We illustrate this on classical $\Sigma$-algebras. These are the algebras for the polynomial set functor

$$
H_{\Sigma} X=\coprod_{\sigma \in \Sigma} X^{n}
$$

where $n$ is the arity of $\sigma$. For every set $X$ (of recursion variables) and every system of mutually recursive equations

$$
x=\sigma\left(x_{1}, \ldots, x_{n}\right),
$$

one for every $x \in X$, where $\sigma \in \Sigma$ has arity $n$ and $x_{i} \in X$, we get the corresponding coalgebra

$$
e: X \longrightarrow H_{\Sigma} X ; \quad x \mapsto\left(x_{1}, \ldots, x_{n}\right) \quad \text { in the } \sigma \text {-summand } X^{n} .
$$

The square (2.1) tells us that the substitution of $e^{\dagger}(x)$ for $x \in X$ makes the formal equations $x=\sigma\left(x_{1}, \ldots, x_{n}\right)$ identities in $A$ :

$$
e^{\dagger}(x)=\sigma^{A}\left(e^{\dagger}\left(x_{1}\right), \ldots, e^{\dagger}\left(x_{n}\right)\right) .
$$

\section{Example 2.3.}

(1) In 21] this concept of corecursive algebras is studied and compared with a number of related concepts. A concrete example of a corecursive algebra from that paper, for the endofunctor $H X=E \times X \times X$ on Set, is the set $E^{\infty}$ of all streams. The operation $a: E \times E^{\infty} \times E^{\infty} \rightarrow E^{\infty}$ is given by $a(e, u, v)$ having head $e$ and continuing by the merge of $u$ and $v$.

(2) If $H$ has a terminal coalgebra $\tau: T \rightarrow H T$, then by Lambek's Lemma $\tau$ is invertible and the resulting algebra $\tau^{-1}: H T \rightarrow T$ is corecursive. In fact, this is the initial corecursive algebra, that is, for every corecursive algebra $(A, a)$ a unique algebra homomorphism from $\left(T, \tau^{-1}\right)$ exists, see the dual of [21, Proposition 2]. There also the converse is proved (dual of Proposition 7), that is, if the initial corecursive algebra exists, then it is a terminal coalgebra (via the inverse of the algebra structure). 
(3) The trivial terminal algebra $H 1 \rightarrow 1$, where 1 is the terminal object in $\mathcal{A}$, is clearly corecursive.

(4) If $a: H A \rightarrow A$ is a corecursive algebra, then so is $H a: H H A \rightarrow H A$, see [21, Proposition 6]. We generalize this in Lemma 2.5 below.

(5) Combining (3) and (4) we conclude that the terminal $\omega^{o p}$-chain

$$
1 \stackrel{a}{\longleftarrow} H 1 \stackrel{H a}{\longleftarrow} H H 1 \stackrel{H H a}{\longleftarrow} \ldots
$$

consists of corecursive algebras. Indeed, the continuation to $H^{i} 1$ for all ordinals (with $H^{i} 1=\lim _{k \leq i} H^{k} 1$ for all limit ordinals) also yields corecursive algebras. This follows from the following.

Proposition 2.4. Let $\mathcal{A}$ be a complete category. Then corecursive algebras are closed under limits in $\mathrm{Alg} H$. Thus, limits of corecursive algebras are formed on the level of $\mathcal{A}$.

Proof. It is easy to verify that limits in Alg $H$ are formed on the level of $\mathcal{A}$. Let us prove that the product of corecursive algebras is corecursive. The proof for general limits is analogous.

Let $(A, a)$ be the product of corecursive algebras $\left(A_{i}, a_{i}\right)$, with projections $p_{i}: A \rightarrow A_{i}$. For every coalgebra $e: X \rightarrow H X$ we have the unique coalgebra-to-algebra morphism $e_{i}^{\dagger}: X \rightarrow A_{i}$, for all $i \in I$, and the morphism $e^{\dagger}=\left\langle e_{i}^{\dagger}\right\rangle: X \rightarrow A=\prod_{i \in I} A_{i}$ is a coalgebrato-algebra morphism. Indeed, for every $i \in I$, the diagram

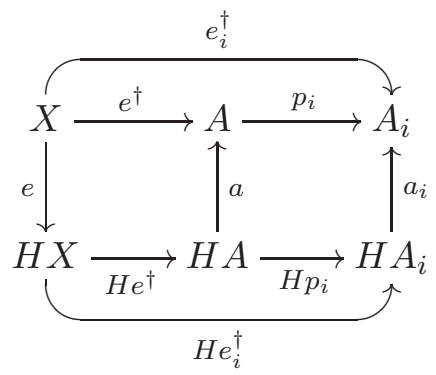

commutes, except perhaps for the left hand inner square; but this suffices to establish the desired commutativity of the left hand square. Since all $A_{i}$ are corecursive, the uniqueness of $e^{\dagger}$ follows from the observation that there is a one-one correspondence between solutions $s: X \rightarrow A$ of $e$ in $A$ and families of solutions $s_{i}: X \rightarrow A_{i}$ of $e$ in $A_{i}$, for all $i \in I$.

In the following we write inl $: X \rightarrow X+Y$ and inr : $Y \rightarrow X+Y$ for the injections of a coproduct.

Lemma 2.5. Let $(A, a)$ be an algebra and $f: B \rightarrow A$ a morphism. Then $(A, a)$ is a corecursive algebra if and only if the algebra

$$
\bar{a} \equiv H(H A+B) \stackrel{H[a, f]}{\longrightarrow} H A \stackrel{\text { inl }}{\longrightarrow} H A+B
$$

is corecursive.

Proof. Let $(A, a)$ be a corecursive algebra and $e: X \rightarrow H X$ be an equation morphism. Then there is a unique solution:

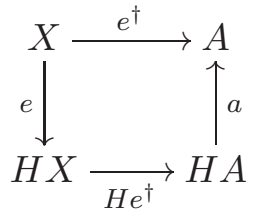


Now inspection of the following commutative diagram shows that inl $\cdot H e^{\dagger} \cdot e: X \rightarrow H A+B$ is a solution of $e$ in $H A+B$.

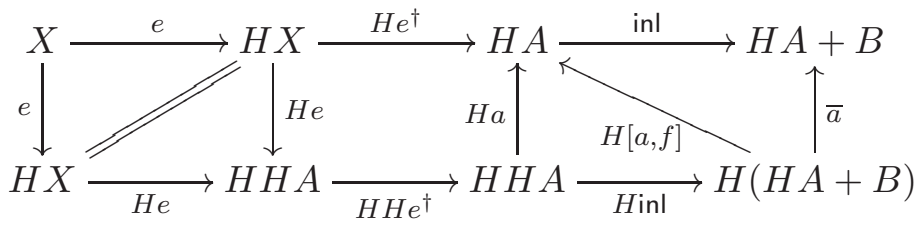

Indeed, commutativity of the middle rectangle follows from Diagram (2.2), the lower triangle on the right is trivial and the upper triangle is the definition of $\bar{a}$. To show the uniqueness of the solution, suppose that $s: X \rightarrow H A+B$ is a solution for $e$, so we have the following commutative diagram:

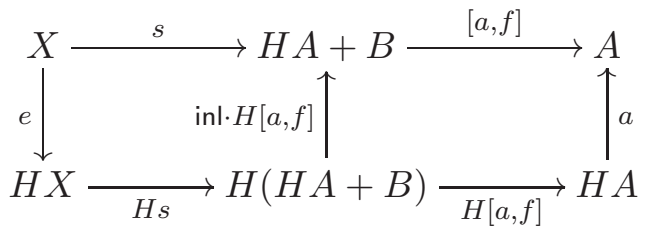

Since the solution $e^{\dagger}$ in $A$ is unique, $e^{\dagger}=[a, f] \cdot s$ and hence

$$
\operatorname{inl} \cdot H e^{\dagger} \cdot e=\operatorname{inl} \cdot H[a, f] \cdot H s \cdot e=s .
$$

Conversely, let $(H A+B$, inl $\cdot H[a, f])$ be a corecursive algebra and $e: X \rightarrow H X$ be an equation morphism. So there exists a unique solution $e^{\dagger}$ of $e$ in $H A+B$, and hence we have the above commutative diagram with $e^{\dagger}$ in lieu of $s$. That is $[a, f] \cdot e^{\dagger}$ is a solution of $e$ in the algebra $(A, a)$. To show uniqueness suppose that $s: X \rightarrow A$ is a solution of $e$, that is $s=a \cdot H s \cdot e$. Then we have Diagrams (2.2) and (2.3) with the morphism $s$ in lieu of $e^{\dagger}$. So, by uniqueness of solution in the corecursive algebra $(H A+B$, inl $\cdot H[a, f])$, we have inl $\cdot H s \cdot e=e^{\dagger}$, and hence $s=a \cdot H s \cdot e=[a, f] \cdot \operatorname{inl} \cdot H s \cdot e=[a, f] \cdot e^{\dagger}$.

Example 2.6. Binary algebras: For $H X=X \times X$, every algebra (given by the binary

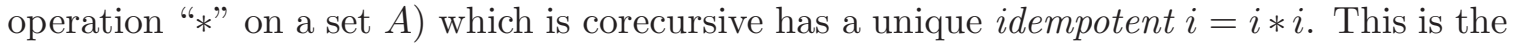
solution of the recursive equation

$$
x=x * x
$$

expressed by the isomorphism $e: 1 \stackrel{\sim}{\rightarrow} 1 \times 1$. Moreover the idempotent is completely factorizable, where the set of all completely factorizable elements is defined to be the largest subset of $A$ such that every element $a$ in it can be factorized as $a=b * c$, with $b, c$ completely factorizable. The corecursiveness of $A$ implies that no other element but $i$ is completely factorizable: consider the system of recursive equations

$$
x_{\epsilon}=x_{0} * x_{1}, \quad x_{0}=x_{00} * x_{01}, \quad \cdots \quad x_{w}=x_{w 0} * x_{w 1}, \quad \ldots
$$

for all finite binary words $w$. Every completely factorizable element $a$ provides a solution $e^{\dagger}$ with $e^{\dagger}\left(x_{\epsilon}\right)=a$. Since solutions are unique, $a=i$.

Conversely, every binary algebra $A$ with an idempotent $i$ which is the only completely factorizable element is corecursive. Indeed, given a morphism $e: X \rightarrow X \times X$, the constant map $e^{\dagger}: X \rightarrow A$ with value $i$ is a coalgebra-to-algebra morphism. Conversely, if $e^{\dagger}$ is a coalgebra-to-algebra morphism, then for every $x \in X$ the element $e^{\dagger}(x)$ is clearly completely factorizable. Therefore, $e^{\dagger}(x)=i$. 
Remark 2.7. Recall the concept of completely iterative algebra (cia for short) from [28]: it is an algebra $(A, a)$ such that for every "flat equation" morphism $e: X \rightarrow H X+A$ there exists a unique solution, i.e. a unique morphism $e^{\dagger}$ such that the square

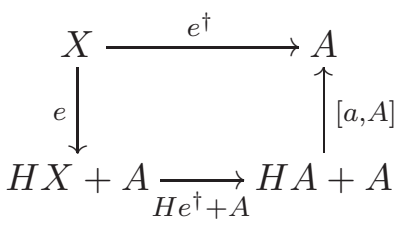

commutes. This is obviously stronger than corecursiveness because every coalgebra $e: X \rightarrow$ $H X$ yields a flat equation morphism inl $\cdot e: X \rightarrow H X+A$. Then solutions are determined uniquely. Thus, for example, in the category of complete metric spaces with distance less than one and nonexpanding functions, all algebras for contracting endofunctors (in the sense of P. America and J. Rutten [17]) are corecursive, because, as proved in [28, they are cia's. Here is a concrete example: $H X=X \times X$ equipped with the metric taking $1 / 2$ of the maximum of the two distances is contracting. Thus every binary algebra whose operation is contracting is corecursive.

Example 2.8. The endofunctor $H X=X \times X$ has many corecursive algebras that are not cia's. For example the algebra $A$ of all binary trees with finitely many leaves. The operation is tree-tupling and the only completely factorizable tree is the complete binary tree $t$. Thus, $A$ is corecursive. However, if $a \in A$ denotes the root-only tree, then the system of recursive equations

$$
\begin{aligned}
& x=x * y \\
& y=a
\end{aligned}
$$

does not have a solution in $A$ (because the tree corresponding to $x$ has infinitely many leaves). Thus $A$ is not a cia.

Lemma 2.9. Every homomorphism $h:(A, a) \rightarrow(B, b)$ in $\operatorname{Alg} H$ with $(A, a)$ and $(B, b)$ corecursive preserves solutions. That is, given a coalgebra $e: X \rightarrow H X$ with a solution $e^{\dagger}: X \rightarrow A$ in the domain algebra, then $h \cdot e^{\dagger}: X \rightarrow B$ is the solution in the codomain one.

Proof. This follows from the diagram

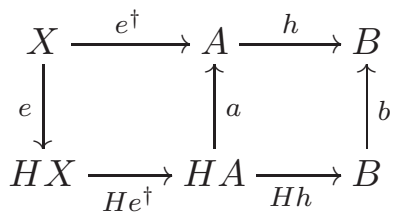

We thus consider corecursive algebras as a full subcategory $\mathrm{Alg}_{C} H$ of $\mathrm{Alg} H$. We obtain a forgetful functor

$$
\operatorname{Alg}_{C} H \quad \rightarrow \mathcal{A} \quad(A, a) \mapsto A
$$

In Section 4 we prove that this forgetful functor has a left adjoint, that is, free corecursive algebras exist, if and only if a terminal coalgebra $T$ exists and every object $Y$ generates a free algebra $F Y$ (i.e., the forgetful functor $\operatorname{Alg} H \rightarrow \mathcal{A}$ has a left adjoint). Our result holds for example for all set functors, and for them the formula for the free corecursive algebra is $T \oplus F Y$, where $\oplus$ is the coproduct in $\mathrm{Alg} H$. 
Recall from [24] that given an infinite cardinal number $\lambda$, a functor is called $\lambda$-accessible if it preserves $\lambda$-filtered colimits. An object $X$ whose hom-functor $\mathcal{A}(X,-)$ is $\lambda$-accessible is called $\lambda$-presentable. A category $\mathcal{A}$ is locally $\lambda$-presentable if it has

(a) colimits, and

(b) a set of $\lambda$-presentable objects whose closure under $\lambda$-filtered colimits is all of $\mathcal{A}$.

A category $\mathcal{A}$ is called locally presentable (and a functor $F$ accessible, resp.) if there exists some $\lambda$ such that $\mathcal{A}$ is locally $\lambda$-presentable (and $F \lambda$-accessible, resp.).

For a $\lambda$-accessible endofunctor $H$, the category $\operatorname{Alg} H$ is also locally $\lambda$-presentable, see [14. For corecursive algebras we have:

Proposition 2.10. Let $\mathcal{A}$ be a locally presentable category. Then for every accessible endofunctor $H$, the category $\operatorname{Alg}_{C} H$ of corecursive algebras is locally presentable.

Proof. Choose an uncountable cardinal number $\lambda$ such that $H$ preserves $\lambda$-filtered colimits and $\mathcal{A}$ is locally $\lambda$-presentable. Then $\lambda$-filtered colimits in Alg $H$ are clearly formed on the level of $\mathcal{A}$. And as proved in [13, every coalgebra is a $\lambda$-filtered colimit of $\lambda$-presentable coalgebras, and these are precisely the coalgebras carried by $\lambda$-presentable objects in $\mathcal{A}$.

By the Reflection Theorem (see [14, Corollary to Theorem 2.48]), in order to establish that the full subcategory $\operatorname{Alg}_{C} H$ of Alg $H$ is locally $\lambda$-presentable, it suffices to see that it is closed in Alg $H$ under limits and $\lambda$-filtered colimits. For limits see Proposition 2.4 and now we prove that $\lambda$-filtered colimits of corecursive algebras in Alg $H$ are corecursive. Indeed, let $\left(A_{t}, a_{t}\right)_{t \in T}$ be a $\lambda$-filtered diagram with colimit $k_{t}:\left(A_{t}, a_{t}\right) \rightarrow(C, c)$. For every coalgebra $e: X \rightarrow H X$, a solution $e^{\dagger}: X \rightarrow A_{t}$ exists in $\left(A_{t}, a_{t}\right)$ and since $k_{t}$ is a homomorphism, $k_{t} \cdot e^{\dagger}$ is a solution in $C$, see Lemma 2.9.

To prove that solutions are unique, assume first that $X$ is $\lambda$-presentable in $\mathcal{A}$. For every solution $e^{\dagger}: X \rightarrow C$ there exists a $t \in T$ such that $e^{\dagger}$ factorizes through $k_{t}$ as follows

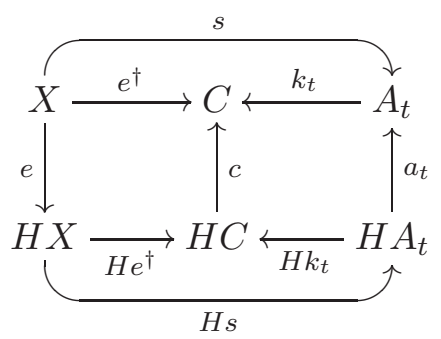

The morphism $k_{t}$ merges $s$ and $a_{t} \cdot H s \cdot e$ :

$$
\begin{aligned}
k_{t} \cdot\left(a_{t} \cdot H s \cdot e\right) & =c \cdot H k_{t} \cdot H s \cdot e \\
& =c \cdot H e^{\dagger} \cdot e \\
& =e^{\dagger} \\
& =k_{t} \cdot s .
\end{aligned}
$$

Consequently, since $k_{t}$ is a colimit morphism of a $\lambda$-filtered colimit, there exists an object $t^{\prime} \in T$ and a connecting morphism $u: A_{t} \rightarrow A_{t^{\prime}}$ which also merges $s$ and $a_{t} \cdot H s \cdot e$, that is

$$
u \cdot s=u \cdot a_{t} \cdot H s \cdot e=a_{t^{\prime}} \cdot H u \cdot H s \cdot e=a_{t^{\prime}} \cdot H(u \cdot s) \cdot e .
$$

This last equation proves that $u \cdot s$ is a solution of $e$ in $A_{t^{\prime}}$, thus $u \cdot s$ is uniquely determined. Hence, $e^{\dagger}$ is uniquely determined from $e^{\dagger}=k_{t} \cdot s=k_{t^{\prime}} \cdot u \cdot s$. 
Next let $X$ be arbitrary. Express $(X, e)$ in the category of coalgebras as a $\lambda$-filtered colimit of coalgebras $\left(X_{i}, e_{i}\right)$ with $X_{i} \lambda$-presentable in $\mathcal{A}$. Let $x_{i}: X_{i} \rightarrow X$ be the corresponding colimit cocone. For every solution $e^{\dagger}: X \rightarrow C$ each $e^{\dagger} \cdot x_{i}$ is a solution of $e_{i}$ since we have the following commutative diagram

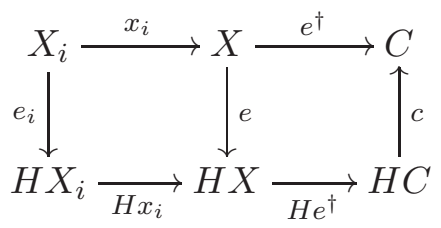

Thus, $e^{\dagger} \cdot x_{i}$ is uniquely determined by the previous case. Since the cocone of all $x_{i}$ 's is collectively epic, this proves that $e^{\dagger}$ is uniquely determined.

Remark 2.11. We obtain from Proposition 2.10 that for an uncountable cardinal number $\lambda$, if $H$ is $\lambda$-accessible and $\mathcal{A}$ locally $\lambda$-presentable, then so is $\operatorname{Alg}_{C} H$. And for $\lambda=\omega$, i.e. $H$ is finitary on the locally finitely presentable category $\mathcal{A}$, we have that $\operatorname{Alg}_{C} H$ is locally $\aleph_{1}$-presentable.

\section{Bloom Algebras}

In the case of iterative algebras, it was proved in [10] that every finitary functor $H$ of $\mathcal{A}$ has a free iterative algebra on every object of $\mathcal{A}$, and the resulting monad $\mathbb{R}$ on $\mathcal{A}$ is a free iterative monad. The next step was a characterization of the Eilenberg-Moore algebras for $\mathbb{R}$ that were called Elgot algebras [9]. An Elgot algebra has for every finitary flat equation $e$ a solution $e^{\dagger}$, but not necessarily unique. Instead, Elgot algebras are equipped with a solution operation $e \mapsto e^{\dagger}$ satisfying some "natural" axioms.

In the present section we take the corresponding step for corecursive algebras. We introduce Bloom algebras as algebras equipped with an operation assigning to every coalgebra $e$ a solution $e^{\dagger}$ which forms a functor. Later we prove that Bloom algebras are (analogously to Elgot algebras) precisely the Eilenberg-Moore algebras for the free corecursive monad, see Theorems 4.12 and 6.4 ,

Definition 3.1. A Bloom algebra is a triple $(A, a, \dagger)$ where $a: H A \rightarrow A$ is an $H$-algebra and $\dagger$ is an operation assigning to every coalgebra $e: X \rightarrow H X$ a coalgebra-to-algebra homomorphism $e^{\dagger}: X \rightarrow A$ so that $\dagger$ is functorial. This means that we obtain a functor

$$
\dagger: \text { Coalg } H \rightarrow \mathcal{A} / A \text {. }
$$

More explicitly, given a coalgebra homomorphism

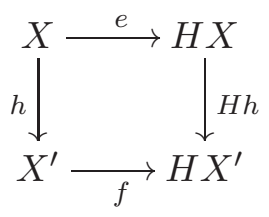

the following triangle commutes

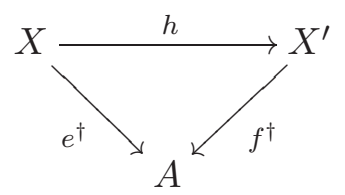




\section{Example 3.2.}

(a) Every corecursive algebra is a Bloom algebra. Indeed, functoriality easily follows from the uniqueness of solutions due to the diagram

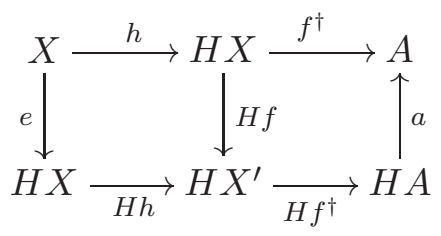

(b) A unary algebra $a: A \rightarrow A(H=I d)$ is a Bloom algebra iff $a$ has a fixpoint, i. e., a morphism $t: 1 \rightarrow A$ with $a \cdot t=t$. More precisely:

(1) Given a fixpoint, then $(A, a, \dagger)$ is a Bloom algebra where $e^{\dagger}=t \cdot$ ! for the unique morphism ! : $X \rightarrow 1$.

(2) Given a Bloom algebra $(A, a, \dagger)$, then $i d_{1}^{\dagger}: 1 \rightarrow A$ is a fixpoint of $a$.

(c) Let $\mathcal{A}$ have finite products. An algebra $a: A \times A \rightarrow A$ for $H X=X \times X$ is a Bloom algebra if and only if it has an idempotent global element, that is $i: 1 \rightarrow A$ satisfying $a \cdot(i \times i)=i$ (recall that $1 \times 1=1)$. More precisely:

(1) Given an idempotent $i$, we have a Bloom algebra $(A, a, \dagger)$, where $\dagger$ is the constant function with value $e^{\dagger}=i \cdot$ !

(2) Given a Bloom algebra $(A, a, \dagger)$, there exists an idempotent $i$ such that $\dagger$ is the constant function with value $e^{\dagger}=i \cdot$ !

Compare this with Example 2.6. In particular every group, considered as a binary algebra in Set, is thus a Bloom algebra in a unique sense. But no nontrivial group is corecursive.

(d) Every continuous algebra is a Bloom algebra if we define $e^{\dagger}$ to be the least solution of $e$. More detailed, let $H$ be a locally continuous endofunctor of the category CPO of complete ordered sets (i. e., partially ordered sets with a least element $\perp$ and with joins of $\omega$-chains). For every $H$-algebra $(A, a)$ and every equation morphism $e: X \rightarrow H X$, we can define in $\operatorname{CPO}(X, A)$ a function $e^{\dagger}: X \rightarrow A$ as a join of the sequence $e_{n}^{\dagger}$ defined by $e_{0}^{\dagger}=$ const $_{\perp}$ and $e_{n+1}^{\dagger}=a \cdot H e_{n}^{\dagger} \cdot e$. Then the least solution of $e$ is $e^{\dagger}=\bigvee_{n<\omega} e_{n}^{\dagger}$ and $(A, a, \dagger)$ is a Bloom algebra. Example (b) demonstrates that this need not be corecursive.

(e) Every product of Bloom algebras is a Bloom algebra. We define $e^{\dagger}=\left\langle e_{i}^{\dagger}\right\rangle$ as in the proof of Proposition 2.4. More generally: every limit of Bloom algebras is a Bloom algebra.

(f) Every complete Elgot algebra in the sense of [8] is a Bloom algebra.

Definition 3.3. By a homomorphism of Bloom algebras from $(A, a, \dagger)$ to $(B, b, \ddagger)$ is meant an algebra homomorphism $h:(A, a) \rightarrow(B, b)$ preserving solutions, that is, for every coalgebra $e: X \rightarrow H X$ the triangle

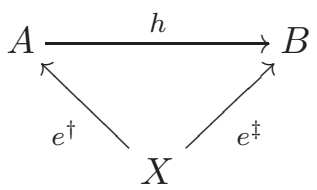

commutes. We denote by $\mathrm{Alg}_{B} H$ the corresponding category of Bloom algebras. 
Proposition 3.4. Let $(T, \tau)$ be a terminal coalgebra for $H$. The category of Bloom algebras for $H$ is isomorphic to the slice category $\left(T, \tau^{-1}\right) / \mathrm{Alg} H$.

Proof. Let us, for a coalgebra $(X, e)$, denote the unique coalgebra homomorphism from $X$ to $T$ by $e^{\sharp}: X \rightarrow T$. We shall define two functors between $\operatorname{Alg}_{B} H$ and $\mathcal{S}=\left(T, \tau^{-1}\right) / \operatorname{Alg} H$ and show that they are mutually inverse.

(a) From Bloom algebras to the slice category $\mathcal{S}$ : given a Bloom algebra $(A, a, \dagger)$ we form the solution $\tau^{\dagger}: T \rightarrow A$ which clearly is an object $\left(A, \tau^{\dagger}\right)$ of $\mathcal{S}$. For a homomorphism $h:(A, a, \dagger) \rightarrow(B, b, \ddagger)$ of Bloom algebras, we clearly have a morphism $h:\left(A, \tau^{\dagger}\right) \rightarrow\left(B, \tau^{\ddagger}\right)$ of $\mathcal{S}$, since $h$ is solution preserving. This defines a functor from $\operatorname{Alg}_{B} H$ to $\mathcal{S}$.

(b) From $\mathcal{S}$ to Bloom algebras: Suppose we are given an object $(A, h)$ in $\mathcal{S}$, that is, an algebra homomorphism:

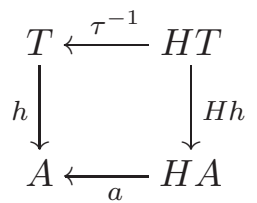

We define for every $e: X \rightarrow H X$ its dagger as $e^{\dagger}=h \cdot e^{\sharp}$. This is functorial; indeed, for every coalgebra homomorphism $k:(X, e) \rightarrow(Y, f)$ we have $f^{\sharp} \cdot k=e^{\sharp}$ by unicity of the universal property of the terminal coalgebra $(T, \tau)$, thus

$$
\begin{aligned}
f^{\dagger} \cdot h & =h \cdot f^{\sharp} \cdot k \\
& =h \cdot e^{\sharp} \\
& =e^{\dagger}
\end{aligned}
$$

In addition, every morphism $m:(A, h) \rightarrow\left(B, h^{\prime}\right)$ of $\mathcal{S}$ is a homomorphism of Bloom algebras $(A, a, \dagger) \rightarrow(B, b, \ddagger):$

$$
\begin{aligned}
m \cdot e^{\dagger} & =m \cdot h \cdot e^{\sharp} \\
& =h^{\prime} \cdot e^{\sharp} \\
& =e^{\ddagger}
\end{aligned}
$$

by definition of $\dagger$

$m$ is a morphism in $\mathcal{S}$

by definition of $\ddagger$

That this gives a functor from $\mathcal{S}$ to $\operatorname{Alg}_{B} H$ is immediate.

(c) The two functors above are mutually inverse. Indeed, it suffices to show that we have a bijection on the level of objects, since both functors are the identity maps on morphisms. So for $(A, h)$ in $\mathcal{S}$ we form first $(A, a, \dagger)$ as in (b) and then $\left(A, \tau^{\dagger}\right)$ as in (a) and we have $\tau^{\dagger}=h \cdot \tau^{\sharp}=h$, as $\tau^{\sharp}$ is the identity (being the unique coalgebra homomorphism from $(T, \tau)$ to itself). Finally, given a Bloom algebra $(A, a, \ddagger)$ we first form $\left(A, \tau^{\ddagger}\right)$ as in (a) and then $(A, a, \dagger)$ as in (b). Then we have

$$
\begin{aligned}
e^{\dagger} & =\tau^{\ddagger} \cdot e^{\sharp} & & \text { by definition of } \dagger \text { in (b) } \\
& =e^{\ddagger} & & \text { by functoriality of } \ddagger
\end{aligned}
$$

This completes the proof. 
Remark 3.5. Being an algebra homomorphism and preserving solutions are independent concepts: neither of them implies the other one. To see this, consider for $H X=X \times X$ an algebra $A=\{a, b\}$ with a binary operation $*$ such that $a$ and $b$ are idempotent. We turn $A$ into a Bloom algebra by taking $e^{\dagger}=$ const $_{a}$ for every $e: X \rightarrow X \times X$. Then there are two homomorphisms from the one-point binary algebra (which clearly is corecursive) to $A$, yet only one of them is solution preserving. Thus, there exist homomorphisms which are not solution preserving.

Conversely, there exist solution preserving morphisms which are not homomorphisms. To see this, let us assume that we have $x * y=a$ for all $x \neq y$ in $A$. There are two different structures of Bloom algebras on $A,\left(A, \alpha, e \mapsto\right.$ const $\left._{a}\right)$ and $\left(A, \alpha, e \mapsto\right.$ const $\left._{b}\right)$. The map on $A$ which swaps $a$ and $b$ is a solution preserving map between the two Bloom algebras, but not a homomorphism.

Proposition 3.6. An initial Bloom algebra is precisely a terminal coalgebra.

More precisely, the statement in Example 2.6 generalizes from corecursive algebras to Bloom algebras. Indeed, the proof in 21] can be used again.

Lemma 3.7. If $(A, a, \dagger)$ is a Bloom algebra and $h:(A, a) \rightarrow(B, b)$ is a homomorphism of algebras, then there is a unique structure of a Bloom algebra on $(B, b)$ such that $h$ is a solution preserving morphism. We call it, the Bloom algebra induced by $h$.

Proof. For every coalgebra $e: X \rightarrow H X$ we define

$$
e^{*} \equiv X \stackrel{e^{\dagger}}{\rightarrow} A \stackrel{h}{\rightarrow} B
$$

and verify that $e^{*}$ is a solution of $e$ by the following commutative diagram

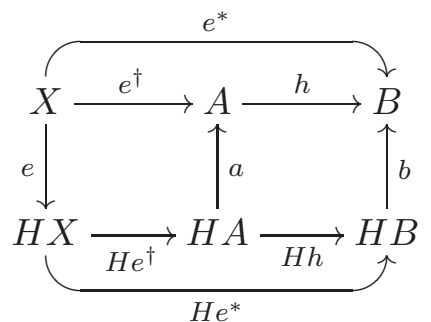

Functoriality is easily checked too: let $g:(X, e) \rightarrow(Y, f)$ be a coalgebra homomorphism. Then the following equations hold:

$$
\begin{aligned}
f^{*} \cdot g & =h \cdot f^{\dagger} \cdot g \\
& =h \cdot e^{\dagger} \\
& =e^{*}
\end{aligned}
$$

by the definition of $(-)^{*}$

by functoriality of $\dagger$

by the definition of $(-)^{*}$

Finally, $h$ is clearly solution preserving.

The unicity of the Bloom algebra structure given by $(-)^{*}$ is clear.

Remark 3.8. We are going to characterize the left adjoint of the forgetful functor

$$
U: \operatorname{Alg}_{B} H \rightarrow \mathcal{A}, \quad(A, a, \dagger) \mapsto A .
$$

In other words, we characterize the free Bloom algebras: they are coproducts $T \oplus F Y$ of the terminal coalgebra and free algebras. For that we first attend to the existence of those ingredients. 
Lemma 3.9. Let $\mathcal{A}$ be a complete category. If $H$ has a free Bloom algebra on an object $Y$ with $\mathcal{A}(Y, H Y) \neq \emptyset$, then $H$ has a terminal coalgebra.

Proof. The free Bloom algebra $(A, a, \dagger)$ on $Y$, is weakly initial in $\mathrm{Alg}_{B} H$. To see this, choose a morphism $e: Y \rightarrow H Y$. For every Bloom algebra $(B, b, \ddagger)$ the solution $e^{\ddagger}: Y \rightarrow B$ extends to a homomorphism $h:(A, a, \dagger) \rightarrow(B, b, \ddagger)$ of Bloom algebras.

Since $\operatorname{Alg}_{B} H$ is complete by Example 3.2 (e), we can use Freyd's Adjoint Functor Theorem. The existence of a weakly initial object implies that $\operatorname{Alg}_{B} H$ has an initial object. Now apply Proposition 3.6

Construction 3.1. Free-Algebra Chain. Recall from [2] that if $\mathcal{A}$ is cocomplete, we can define a chain constructing the free $H$-algebra on $Y$ as follows:

$$
Y \stackrel{\mathrm{inr}}{\longrightarrow} H Y+Y \stackrel{H \mathrm{inr}+Y}{\longrightarrow} H(H Y+Y)+Y \longrightarrow \ldots
$$

We mean the essentially unique chain $V: \operatorname{Ord} \rightarrow \mathcal{A}$ with

$$
\begin{aligned}
V_{0} & =Y \\
V_{i+1} & =H V_{i}+Y
\end{aligned}
$$

and for limit ordinals $i$

$$
V_{j}=\underset{k<j}{\operatorname{colim}} V_{k}
$$

whose connecting morphisms $v_{i, j}: V_{i} \rightarrow V_{j}$ are defined by

$$
v_{0,1} \equiv \operatorname{inr}: Y \rightarrow H Y+Y \quad \text { and } \quad v_{i+1, j+1} \equiv H v_{i, j}+Y
$$

and for limit ordinals $j$

$$
\left(v_{k, j}\right)_{k<j} \text { is the colimit cocone. }
$$

This chain is called the free-algebra chain. If it converges at some ordinal $\lambda$, that is, if $v_{\lambda, \lambda+1}$ is an isomorphism, then $V_{\lambda}$ is a free algebra on $Y$. More detailed: this isomorphism turns $V_{\lambda}$ into a coproduct

$$
V_{\lambda}=H V_{\lambda}+Y
$$

and thus $V_{\lambda}$ is an algebra via the left-hand coproduct injection, and the right-hand one $Y \rightarrow V_{\lambda}$ yields the universal arrow.

Corollary 3.10. Every accessible endofunctor of a cocomplete category has free algebras.

Indeed, if the given functor is $\lambda$-accessible, the free-algebra chain converges at $\lambda$.

Definition 3.11. (See [15]) We say that in a given category monomorphisms are constructive provided that

(a) if $m_{i}: A_{i} \rightarrow B_{i}$ are monomorphisms for $i=1,2$ then $m_{1}+m_{2}: A_{1}+A_{2} \rightarrow B_{1}+B_{2}$ is a monomorphism,

(b) coproduct injections are monomorphisms, and

(c) if $a_{i}: A_{i} \rightarrow A,(i<\lambda)$, is a colimit of an $\lambda$-chain and $m: A \rightarrow B$ has all composites $m \cdot a_{i}$ monic, then $m$ is monic.

Example 3.12. Sets, posets, graphs, abelian groups have constructive monomorphisms. If $\mathcal{A}$ has constructive monomorphisms, then all functor categories $\mathcal{A}^{\mathcal{C}}$ do. In all locally finitely presentable categories condition (c) holds (see [14), but (a) and (b) can fail. 
Proposition 3.13. Let $\mathcal{A}$ be a cocomplete, wellpowered category with constructive monomorphisms. If $H$ has a free Bloom algebra on $Y$ and preserves monomorphisms, then it also has a free algebra on $Y$.

Proof. Let $(A, a, \dagger)$ be a free Bloom algebra and $\eta: Y \rightarrow A$ be the universal arrow.

(1) We prove that $A=H A+Y$ with coproduct injection $a$ and $\eta$. The algebra

$$
b \equiv H(H A+Y) \stackrel{H[a, \eta]}{\longrightarrow} H A \stackrel{\text { inl }}{\longrightarrow} H A+Y
$$

is a Bloom algebra when we put, as in Lemma 2.5,

$$
e^{\ddagger}=\mathrm{inl} \cdot H e^{\dagger} \cdot e, \quad \text { for all } e: X \rightarrow H X .
$$

Indeed, functoriality is easy to verify. Consequently, there exists a unique solution preserving homomorphism

$$
h: A \rightarrow H A+Y \text { with } h \cdot \eta=\text { inr. }
$$

We prove that $h$ is inverse to $[a, \eta]$. For that, observe that $[a, \eta]$ is also a homomorphism

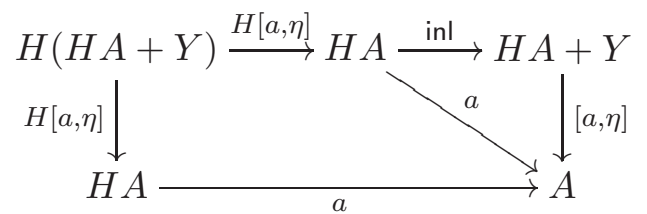

and also preserves solutions:

$$
[a, \eta] \cdot e^{\ddagger}=a \cdot H e^{\dagger} \cdot e=e^{\dagger} .
$$

The composite with $h$ yields an endomorphism of $(A, a, \dagger)$ such that

$$
([a, \eta] \cdot h) \cdot \eta=[a, \eta] \cdot \operatorname{inr}=\eta \text {. }
$$

The universal property thus implies

$$
[a, \eta] \cdot h=i d_{A}
$$

Since $h$ is a homomorphism,

$$
h \cdot a=b \cdot H h=\mathrm{inl} \cdot H([a, \eta] \cdot h)=\mathrm{inl} .
$$

We conclude that $h$ is inverse to $[a, \eta]$ :

$$
h \cdot[a, \eta]=[h \cdot a, h \cdot \eta]=[\mathrm{inl}, \mathrm{inr}]=i d .
$$

Therefore, $A=H A+Y$ with coproduct injections $a$ and $\eta$.

(2) We define a cone $m_{i}: V_{i} \rightarrow A$ of the free-algebra chain by

$$
m_{0} \equiv \eta: Y \rightarrow A
$$

and

$$
m_{i+1} \equiv H V_{i}+Y \stackrel{H m_{i}+i d}{\longrightarrow} H A+Y \stackrel{[a, \eta]}{\longrightarrow} A .
$$

More precisely: there is a unique cone with the above properties. The verification of $m_{i+1} \cdot v_{i, i+1}=m_{i}$ is an easy transfinite induction on $i$, and the limit steps then follow automatically from $V_{i}=\operatorname{colim}_{k<i} V_{k}$. Moreover, since monomorphisms are constructive, all $m_{i}$ 's are monomorphisms: $m_{0}$ is a coproduct injection of $A=H A+Y$, if $m_{i}$ is a monomorphism, then so is $H m_{i}+i d$, hence, so is $m_{i+1}$, and the limit steps are clear. Since $A$ has only a set of subobjects, there exist $j>i$ such that $m_{i}$ and $m_{j}$ represent the 
same subobject, i. e., $v_{i, j}$ is an isomorphism. Then also $v_{i+1, j+1}$ is an isomorphism with an inverse $v^{\prime}$, say. It follows that $v_{j, j+1}$ is an isomorphism, too: it is monomorphic since $m_{j+1} \cdot v_{j, j+1}=m_{j}$, and it is a split epimorphism since $v_{j, j+1} \cdot v_{i+1, j} \cdot v^{\prime}=v_{i+1, j+1} \cdot v^{\prime}=i d$. Therefore, $V_{j}$ is a free algebra on $Y$.

Corollary 3.14. If a set functor has a free Bloom algebra on $Y$, it has both a terminal coalgebra $T$ and a free algebra $F Y$ on $Y$.

Proof. For the existence of $T$ use Lemma 3.9. for $\mathcal{A}=$ Set we have $\mathcal{A}(Y, H Y) \neq \emptyset$ whenever $H Y \neq \emptyset$ or $Y=\emptyset$, and in the remaining case where $Y \neq \emptyset$ and $H Y=\emptyset, T$ trivially exists since one deduces that $H$ is the constant functor on $\emptyset$; indeed, for an any set $X$ pick some $f: X \rightarrow Y$, then $H f: H X \rightarrow H Y=\emptyset$ implies $H X=\emptyset$.

For the existence of $F Y$ apply Proposition 3.13 in the case where $H$ preserves monomorphisms. If it does not, redefine it in $\emptyset$ and obtain a set functor $H^{\prime}$ with $H^{\prime} X=H X$, for all $X \neq \emptyset$ and $H^{\prime} \emptyset=\emptyset$. Then $H^{\prime}$ preserves monomorphisms and, whenever $Y \neq \emptyset$, it has a free Bloom algebra on $Y$ if and only if $H$ does. The case $Y=\emptyset$ is obtained from Proposition 3.6 .

We are going to characterize free Bloom algebras. From Lemma 3.9 and Proposition 3.13 we know that the assumption that $T$ and $F Y$ exist is "natural". Since the terminal coalgebra $\tau: T \rightarrow H T$ has by Lambek's Lemma an invertible structure map $\tau$, we can view it as an algebra. In the next proposition we assume that the coproduct of $T$ and $F Y$ exists in Alg $H$. In Section 4 we will see that this actually follows from the existence of a free Bloom algebra.

Notation 3.15. Coproducts in Alg $H$ are denoted by $(A, a) \oplus(B, b)$.

Theorem 3.16. Suppose that $H$ has a terminal coalgebra $T$, a free algebra $F Y$ on $Y$ and their coproduct $T \oplus F Y$. Then the last algebra is the free Bloom algebra on $Y$.

Remark 3.17. More precisely, let $\eta: Y \rightarrow F Y$ be the universal arrow of the free algebra $F Y$. Take the unique Bloom algebra structure $\dagger$ on $T \oplus F Y$ induced by inl $: T \rightarrow T \oplus F Y$ following Lemma 3.7. Then this forms the free Bloom algebra $(T \oplus F Y, a, \dagger)$ on $Y$ with the universal arrow inr $\cdot \eta$. The latter means that for every Bloom algebra $(B, b, \ddagger)$ and every morphism $g: Y \rightarrow B$ in $\mathcal{A}$ there exists a unique homomorphism of Bloom algebras $h: T \oplus F Y \rightarrow B$ such that $h \cdot$ inr $\cdot \eta=g$.

Proof. Given a Bloom algebra $(B, b, \ddagger)$ and morphism $g: Y \rightarrow B$, we obtain a unique homomorphism $\bar{g}:\left(F Y, \varphi_{Y}\right) \rightarrow(B, b)$ with $g=\bar{g} \cdot \eta$. We also have a unique solutionpreserving homomorphism $f:\left(T, \tau^{-1}, \dagger\right) \rightarrow(B, b, \ddagger)$, see Proposition 3.6. This yields a homomorphism $[f, \bar{g}]: T \oplus F Y \rightarrow B$ which is clearly solution-preserving: recall from Lemma 3.7 that solutions in $T \oplus F Y$ have the form inl $\cdot e^{\dagger}$. Thus, $[f, \bar{g}] \cdot\left(\right.$ inl $\left.\cdot e^{\dagger}\right)=f \cdot e^{\dagger}=e^{\ddagger}$. And this is the desired morphism since $[f, g] \cdot \operatorname{inr} \cdot \eta=\bar{g} \cdot \eta=g$.

Conversely, given a solution-preserving homomorphism $h: T \oplus F Y \rightarrow B$ with $h \cdot$ inr $\cdot \eta=$ $g$, then $h=[f, \bar{g}]$, because $h \cdot \mathrm{inl}: T \rightarrow B$ is clearly solution-preserving, hence $h \cdot \mathrm{inl}=f$. Also $h \cdot \mathrm{inr}$ is a homomorphism from $F Y$ with $h \cdot \mathrm{inr} \cdot \eta=g$, thus $h \cdot \mathrm{inr}=\bar{g}$.

Corollary 3.18. Every accessible endofunctor of a locally presentable category has free Bloom algebras. They have the form $T \oplus F Y$.

Indeed, recall that the assumptions mean that there exists an infinite ordinal $\lambda$ such that 
(a) $\mathcal{A}$ is cocomplete and has a set $\mathcal{A}_{\lambda}$ of $\lambda$-presentable objects (that is, objects whose homfunctors preserve $\lambda$-filtered colimits) such that every object is a $\lambda$-filtered colimit of objects in $\mathcal{A}_{\lambda}$, and

(b) $H$ preserves $\lambda$-filtered colimits.

From this it follows that Coalg $H$ is locally $\bar{\lambda}$-presentable, where $\bar{\lambda}=\max \left\{\lambda, \aleph_{1}\right\}$ (see [13]). Thus, this category has a terminal object, $T$. We know from Corollary 3.10 that the free algebra $F Y$ exists. And $T \oplus F Y$ exists since the category of algebras for an accessible functor on a locally presentable category is itself locally presentable and therefore comcomplete.

Remark 3.19. For concrete examples of $T \oplus F Y$ see Example 4.7 below.

Proposition 3.20. Let $\mathcal{A}$ be a complete category. Then so is $\operatorname{Alg}_{B} H$ and limits of Bloom algebras are formed on the level of $\mathcal{A}$.

Proof. This is completely analogous to the proof of Proposition 2.4. The verification that the function $e \mapsto\left\langle e_{i}^{\dagger}\right\rangle$ is functorial is trivial.

Corollary 3.21. For a complete category $\mathcal{A}$, the monomorphisms in $\mathrm{Alg}_{B} H$ are precisely those homomorphisms carried by monomorphisms in $\mathcal{A}$.

Proof. To see this use that in any category with pullbacks a morhism $m$ is a monomorphism iff its kernel pair consists of two identity morphisms.

\section{Free Corecursive Algebras}

For accessible functors $H$ we prove that free corecursive algebras $M Y$ exist and, in the case where $H$ preserves monomorphisms, they coincide with the free Bloom algebras $M Y=$ $T \oplus F Y$. Moreover an iterative construction of these free algebras (closely related to the free algebra chain in 3.1) is presented.

We first prove that the category of corecursive algebras is strongly epireflective in the category of Bloom algebras. That is, the full embedding is a right adjoint, and the components of the unit of the adjunction are strong epimorphisms.

Proposition 4.1. For every accessible endofunctor of a locally presentable category, corecursive algebras form a strongly epireflective subcategory of the category of Bloom algebras. In particular, every Bloom subalgebra of a corecursive algebra in $\operatorname{Alg}_{B} H$ is corecursive.

Proof. Let $\lambda$ be an infinite cardinal such that $\mathcal{A}$ is a locally $\lambda$-presentable category and $H$ preserves $\lambda$-filtered colimits. Since $\lambda$ can be chosen arbitrarily large, we can assume that $\lambda$ is uncountable. The category $\operatorname{Alg}_{B} H$ is locally $\lambda$-presentable. The proof is analogous to that of Proposition 2.10 (the only difference is that in the proof of the uniqueness of $e^{\dagger}$ we simply observe that since $k_{t}$ 's are supposed to be solution-preserving, we have $e^{\dagger}=k_{t} \cdot s$, where $s$ is the dagger of $e$ in $A_{t}$ ). Consequently, $\operatorname{Alg}_{B} H$ is a complete, well-powered, and cowellpowered category, and it has (strong epi-mono) factorization of morphisms, see 14. The full subcategory of corecursive algebras is closed under products by Proposition 2.4. Thus, the proof will be completed when we prove that the subcategory of corecursive algebras is closed in $\mathrm{Alg}_{B} H$ under subalgebras, then it is strongly epireflective (see [5, Theorem 16.8]). 
Let $m:(A, a, \dagger) \rightarrow(B, b, \ddagger)$ be a monomorphism in $\operatorname{Alg}_{B} H$ with $(B, b, \ddagger)$ corecursive. From Corollary 3.21 we have that $m$ is a monomorphism in $\mathcal{A}$. It is our task to prove that for every coalgebra $e: X \rightarrow H X$ the morphism $e^{\dagger}$ is the unique solution in $A$. This follows from the fact that $m \cdot e^{\dagger}=e^{\ddagger}$. Since $e^{\ddagger}$ is unique in $B$ and $m$ is a monomorphism in $\mathcal{A}$, the proof is concluded.

Corollary 4.2. Every accessible endofunctor of a locally presentable category has free corecursive algebras.

Indeed, since the functors $\operatorname{Alg}_{C} H \hookrightarrow \operatorname{Alg}_{B} H$ and $\operatorname{Alg}_{B} H \rightarrow \mathcal{A}$ have left adjoints by Corollary 3.18 and Proposition 4.1, their composite has a left adjoint.

Remark 4.3. We believe that in the generality of the above corollary, the free corecursive algebras are $T \oplus F Y$ (as in Corollary 3.18). But we can only prove this in the case where $H$ preserves monomorphisms and monomorphisms are constructive. We are going to apply the following transfinite construction of free corecursive algebras closely related to the free algebra construction of 3.1

Construction 4.1. Free-Corecursive-Algebra Chain.

Let $\mathcal{A}$ be cocomplete and $H$ have a terminal coalgebra $(T, \tau)$. We define an essentially unique chain $U:$ Ord $\rightarrow \mathcal{A}$ by

$$
\begin{aligned}
U_{0} & =T \\
U_{i+1} & =H U_{i}+Y
\end{aligned}
$$

and for limit ordinals $j$

$$
U_{j}=\operatorname{colim}_{k<j} U_{k}
$$

The connecting morphisms $u_{i, j}: U_{i} \rightarrow U_{j}$ are defined by

$$
\begin{gathered}
u_{0,1} \equiv T \stackrel{\tau}{\rightarrow} H T \stackrel{\text { inl }}{\rightarrow} H T+Y \\
u_{i+1, j+1}=H u_{i, j}+i d_{Y}
\end{gathered}
$$

and for limit ordinals $j$

$$
\left(u_{k, j}\right)_{k<j} \text { is the colimit cocone. }
$$

We say that the chain converges at $\lambda$ if the connecting morphism $u_{\lambda, \lambda+1}$ is an isomorphism, thus $U_{\lambda}=H U_{\lambda}+Y$. Then $U_{\lambda}$ is an algebra (via inl) connected to $Y$ via inr.

Proposition 4.4. Let $\mathcal{A}$ be a cocomplete and wellpowered category with constructive monomorphisms, and let $H$ preserve monomorphisms and have a terminal coalgebra $T$. If the corecursive chain for $Y$ converges in $\lambda$ steps, then $U_{\lambda}=T \oplus F Y$.

Remark 4.5. (a) More detailed, we prove that a free algebra $F Y$ exists and the algebra inl : $H U_{\lambda} \rightarrow U_{\lambda}$ (obtained from $U_{\lambda}=H U_{\lambda}+Y$ ) is a coproduct of $T$ and $F Y$ in Alg $H$.

(b) The proposition is valid for every fixpoint of $H$, not necessarily a terminal coalgebra: given any isomorphism $\tau: T \rightarrow H T$ and forming the corresponding chain with $U_{0}=T$ and $U_{i+1}=H U_{i}+Y$, then whenever it converges, it yields a coproduct of $\left(T, \tau^{-1}\right)$ and the free algebra on $Y$ in $\operatorname{Alg} H$. 
Proof. (1) A free algebra $F Y$ exists. To prove this, we define a natural transformation $\left(m_{i}: V_{i} \rightarrow U_{1+i}\right)_{i \in \text { Ord }}$ from the free-algebra chain to the corecursive chain (delayed on finite ordinals by one step, recall that $1+i=i$ for all infinite ordinals). Put

$$
m_{0}=\mathrm{inr}: Y \rightarrow H T+Y
$$

and

$$
m_{i+1}=H m_{i}+i d_{Y}: H V_{i}+Y \rightarrow H U_{1+i}+Y .
$$

The first naturality square

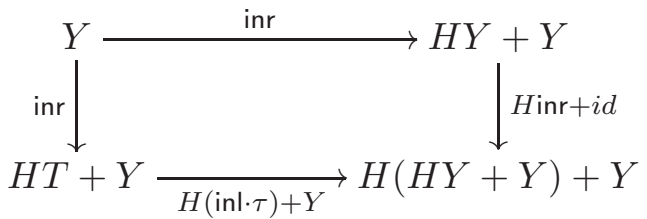

clearly commutes, and the $i$-th square implies the next one easily. Therefore, the limit ordinals $i$ define $m_{i}$ automatically. Since $H$ preserves monomorphisms, we see by transfinite induction that all $m_{i}$ 's are monic. Consequently, we obtain a transfinite chain of subobjects of $U_{\lambda}$

$$
V_{i} \stackrel{m_{i}}{\longrightarrow} U_{1+i} \stackrel{u_{\lambda, 1+i}^{-1}}{\longrightarrow} U_{\lambda}, \quad \text { for } i \geq \lambda .
$$

Since $U_{\lambda}$ has only a set of subobjects, there exist ordinals $\sigma$ and $\rho$ with $\rho>\sigma \geq \lambda$ such that above monomorphisms with $i=\rho$ and $i=\sigma$ represent the same subobject. Similar reasoning as in point (2) of the proof of Proposition 3.13 then shows that $v_{\rho, \rho+1}$ is an isomorphism using the commutative triangle

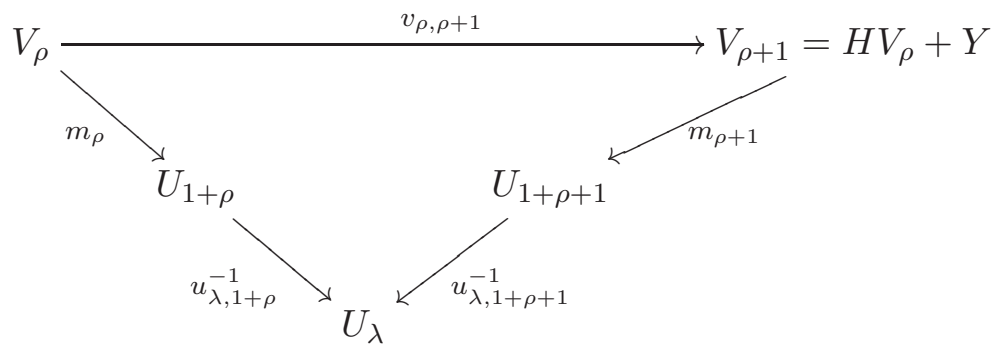

We thus proved that the algebra $\left(V_{\rho}\right.$, inl $)$ is free on $Y$ with respect to inr $: Y \rightarrow V_{\rho}$. Shortly $F Y=V_{\rho}$.

(2) Analogously, $U_{\lambda}$ is an algebra with respect to inl :HU $H U_{\lambda} \rightarrow U_{\lambda}$ (since $u_{\lambda, \lambda+1}$ is invertible). We will prove that this algebra is the coproduct of $T$ and $F Y$ with injections

$$
\overline{\text { inl }} \equiv u_{0, \lambda}: T \rightarrow U_{\lambda}
$$

and

$$
\overline{\mathrm{inr}} \equiv F Y=V_{\rho} \stackrel{m_{\rho}}{\longrightarrow} U_{1+\rho} \stackrel{u_{\lambda, 1+\rho}^{-1}}{\longrightarrow} U_{\lambda}
$$


in Alg $H$. Indeed, $\overline{\mathrm{inl}}$ is an algebra homomorphism: $\overline{\mathrm{inl}}=u_{1, \lambda} \cdot u_{0,1}$ and we have $u_{1, \lambda+1}=$ $H u_{0, \lambda}+Y$, therefore the following square commutes:

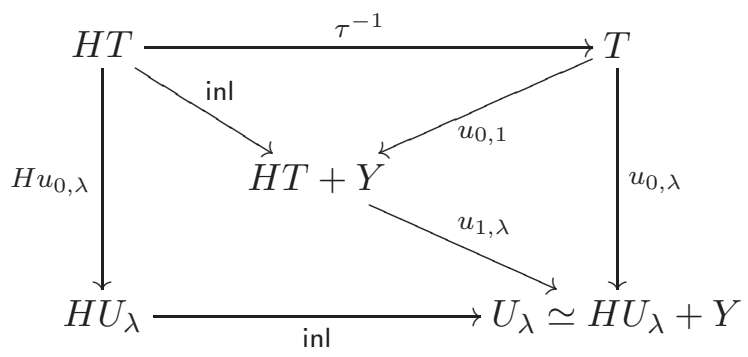

Also inr is a homomorphism: the following diagram

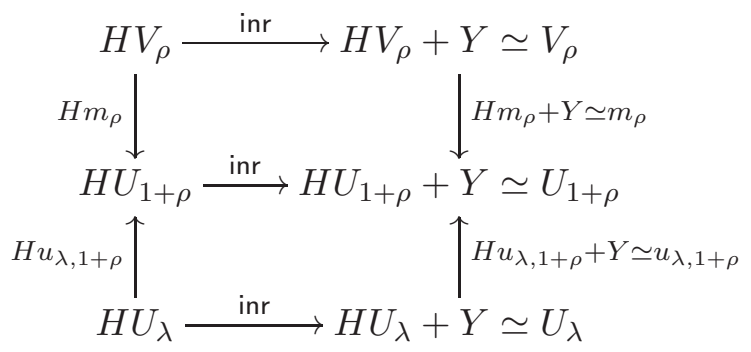

commutes and yields (by inverting $u_{\lambda, 1+\rho}$ ) the square

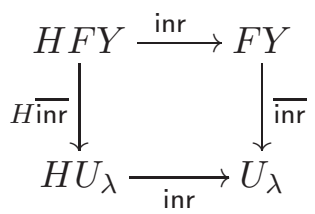

To verify the universal property, let $b: H B \rightarrow B$ be an algebra and $f: T \rightarrow B$ and $g: F Y \rightarrow B$ be homomorphisms. We prove that there exists a unique homomorphism $h: U_{\lambda} \rightarrow B$ with $f=h \cdot \overline{\text { inl }}$ and $g=h \cdot \overline{\text { inr. Put }} \hat{g}=g \cdot$ inr $: Y \rightarrow B$.

Existence: Define a cocone $h_{i}: U_{i} \rightarrow B$ of the chain from Construction 4.1 by

$$
h_{0}=f, \quad \text { and } \quad h_{i+1} \equiv H U_{i}+Y \stackrel{H h_{i}+Y}{\longrightarrow} H B+Y \stackrel{[b, \hat{g}]}{\longrightarrow} B
$$

The first naturality triangle commutes because $f$ is a homomorphism, thus, $f=b \cdot H f \cdot \tau$ :

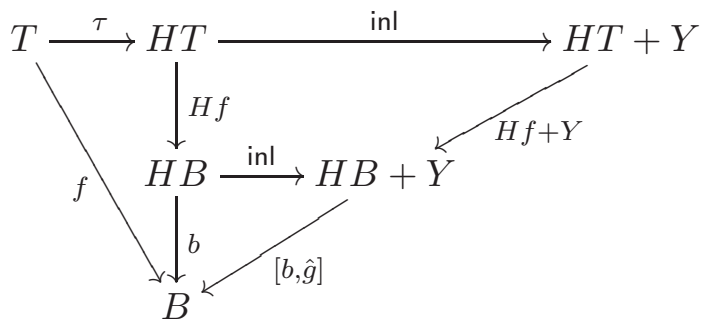

The further verification of the naturality, $h_{i}=h_{i+1} \cdot u_{i+1}$, is now an easy transfinite induction. We obtain the desired homomorphism $h_{\lambda}: U_{\lambda} \rightarrow B$. Indeed, since $h_{\lambda+1} \cdot \operatorname{inl}=b \cdot H h_{\lambda}$ (by 
the above rule for $h_{i+1}$ ), the square

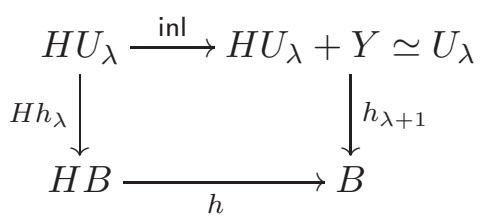

commutes.

The first equation $f=h_{\lambda} \cdot \overline{\text { inl }}$ follows from inl $=u_{0, \lambda}$ and $h_{\lambda} \cdot u_{0, \lambda}=h_{0}=f$. For the second one $g=h_{\lambda} \cdot \overline{\mathrm{inr}}: F Y \rightarrow B$ observe that both sides are algebra homomorphisms. Thus, it is sufficient to prove that the universal arrow inr : $Y \rightarrow V_{\rho}\left(\simeq H V_{\rho}+Y\right)$ merges them. Recall that $\overline{\operatorname{inr}}=u_{\lambda, 1+\rho}^{-1} \cdot m_{\rho}$, thus we need to prove

$$
\hat{g}=h_{\lambda} \cdot \overline{\mathrm{inr}} \cdot \mathrm{inr}=h_{1+\rho} \cdot m_{\rho} \cdot \mathrm{inr} .
$$

This follows from $h_{1+\rho+1}=[b, \hat{g}] \cdot\left(H h_{1+\rho}+Y\right)=\left[b \cdot H h_{1+\rho}, \hat{g}\right]$, thus the outside of the diagram below commutes as desired:

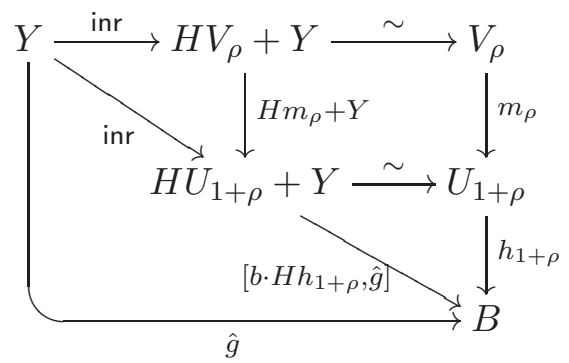

Uniqueness: Consider an algebra homomorphism $h: U_{\lambda} \rightarrow B$ with $h \cdot \overline{\text { inl }}=f$ and $h \cdot \overline{\operatorname{inr}}=g$, we prove $h \cdot u_{i, \lambda}=h_{i}$ by transfinite induction on $i \leq \lambda$. The case $i=\lambda$ yields $h=h_{\lambda}$. The initial step is clear:

$$
h \cdot u_{0, \rho}=h \cdot \overline{\mathrm{inl}}=f=h_{0} .
$$

Assuming $h \cdot u_{i, \lambda}=h_{i}$, we are going to prove that the triangle

$$
\begin{gathered}
H U_{i}+Y=U_{i+1} \\
H u_{i, \lambda}+Y \simeq u_{i+1, \lambda} \downarrow \\
H U_{\lambda}+Y \simeq U_{\lambda} \longrightarrow h
\end{gathered}
$$

commutes. The left-hand component with domain $H U_{i}$ commutes because $h$ is a homomorphism, that is, $h \cdot \mathrm{inl}=b \cdot H h$ :

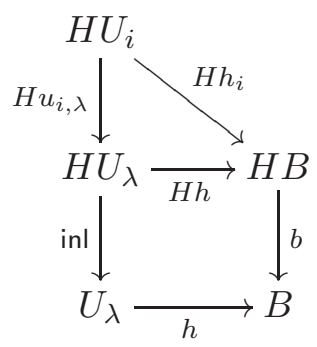


The right-hand one with domain $Y$ follows from $g=h \cdot \overline{\operatorname{inr}}=h \cdot u_{\lambda, 1+\rho}^{-1} \cdot m_{\rho}$ : we have

$$
\hat{g}=g \cdot \operatorname{inr}=h \cdot u_{\lambda, 1+\rho}^{-1} \cdot m_{\rho} \cdot \operatorname{inr}=h \cdot u_{i+1, \lambda} \cdot \mathrm{inr},
$$

where the last equation follows from the commutative diagram below expressing $u_{i+1, \lambda+1}=$ $H u_{i, \lambda}+Y$ :

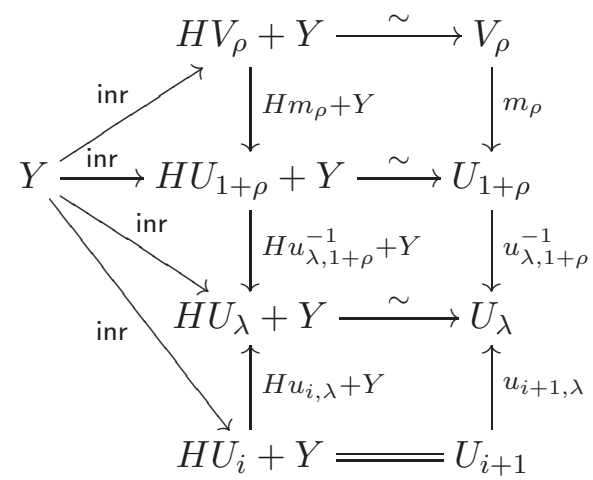

Finally, for a limit ordinal $\alpha$ we easily derive $h \cdot u_{\alpha, \lambda}=h_{\alpha}$ by extending with the colimit injections $u_{i, \alpha}, i \leq \alpha$, and using that they are jointly epic:

$$
h \cdot u_{\alpha, \lambda} \cdot u_{i, \alpha}=h \cdot u_{i, \lambda}=h_{i}=h_{\alpha} \cdot u_{i, \alpha} .
$$

Theorem 4.6. Let $\mathcal{A}$ be a locally presentable category with constructive monomorphisms. Every accessible endofunctor preserving monomorphisms has free corecursive algebras $M Y=$ $T \oplus F Y$.

Proof. From Corollary 3.18 we know that $T \oplus F Y$ is a free Bloom algebra, thus, it is sufficient to prove that this algebra is corecursive. For that, we use Proposition 4.1 and find a corecursive algebra such that $T \oplus F Y$ is its subalgebra; this will finish the proof.

The endofunctor $H(-)+Y$ is also accessible. Thus, it also has a terminal coalgebra (see the proof of Corollary [3.18). We denote it by $T Y$. The components of the inverse of its coalgebra structure $T Y \stackrel{\sim}{\rightarrow} H(T Y)+Y$ are denoted by $\tau_{Y}: H(T Y) \rightarrow T Y$ and $\eta_{Y}: Y \rightarrow T Y$, respectively. As proved in [28] the algebra $T Y$ is a cia for $H$, cf. Remark 2.7. We are going to prove that $T \oplus F Y$ is a subalgebra of this $H$-algebra $T Y$.

Since $H$ is accessible and preserves monomorphisms, the terminal chain of $H$ converges (and yields a terminal coalgebra $T$ ), as proved in [16]. That is, if we define an chain $W: \operatorname{Ord}^{o p} \rightarrow \mathcal{A}$ on objects by

$$
W_{0}=1 \quad \text { and } \quad W_{i+1}=H W_{i}
$$

with $W_{i}=\lim _{k<i} W_{k}$ for limit ordinals (and on morphisms by $w_{1,0}: H 1 \rightarrow 1$ unique, $w_{i+1, j+1}=H w_{i, j}$ and $\left(w_{i, k}\right)_{k<i}$ forming a limit cone), then there exists an ordinal $\lambda$ such that $w_{\lambda+1, \lambda}: H W_{\lambda} \rightarrow W_{\lambda}$ is invertible. We then get

$$
T=W_{\lambda} \quad \text { and } \quad \tau=w_{\lambda+1, \lambda}^{-1} .
$$

We can choose $\lambda$ arbitrarily large, thus we can assume that $\lambda$ is a cardinal such that $H$ is $\lambda$-accessible.

Since monomorphisms are constructive, $H(-)+Y$ also preserves monomorphisms and is also $\lambda$-accessible. Denote by $\bar{W}: \operatorname{Ord}^{o p} \rightarrow \mathcal{A}$ its terminal chain. Then this chain converges and yields a terminal coalgebra $T Y$. Since we again can choose an arbitrary large ordinal 
for the convergence of $\bar{W}$, we can assume that this is the above cardinal $\lambda$, thus, $T Y=\bar{W}_{\lambda}$ and $\left[\tau_{Y}, \eta_{Y}\right]=\bar{w}_{\lambda+1, \lambda}: H(T Y)+Y \rightarrow T Y$. We conclude that $T$ is a (canonical) subalgebra of $T Y$ : define a natural transformation $m_{i}: W_{i} \rightarrow \bar{W}_{i}$ by $m_{0}=i d_{1}$ and $m_{i+1} \equiv H W_{i} \stackrel{H m_{i}}{\longrightarrow}$ $H \bar{W}_{i} \stackrel{\text { inl }}{\longrightarrow} H \bar{W}_{i}+Y$. The constructivity of monomorphisms implies that inl is monic, thus, we see by easy transfinite induction that $m_{i}$ 's are monomorphisms for all $i \in$ Ord. And $m_{\lambda}: T \rightarrow T Y$ is a coalgebra homomorphism because the $\lambda$-th naturality square of $\left(m_{i}\right)$ yields $m_{\lambda} \cdot \tau=\tau_{Y} \cdot H m_{\lambda}$ :

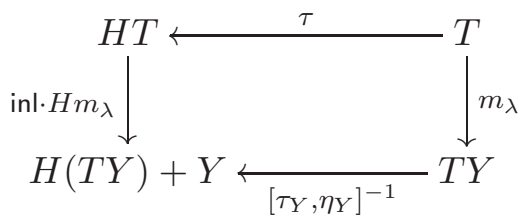

We now define a cocone $p_{i}: U_{i} \rightarrow T Y$, for $i \leq \lambda$, of the chain from Construction 4.1 by

$$
p_{0}=m_{\lambda}: T \rightarrow T Y
$$

and

$$
p_{i+1} \equiv H U_{i}+Y \stackrel{H p_{i}+Y}{\longrightarrow} H(T Y)+Y \stackrel{\left[\tau_{Y}, \eta_{Y}\right]}{\longrightarrow} T Y .
$$

We need to verify compatibility, $p_{i}=p_{i+1} \cdot u_{i, i+1}$, from which the limit steps follow automatically. For $i=0$, use that $m_{\lambda}$ is a coalgebra homomorphism:

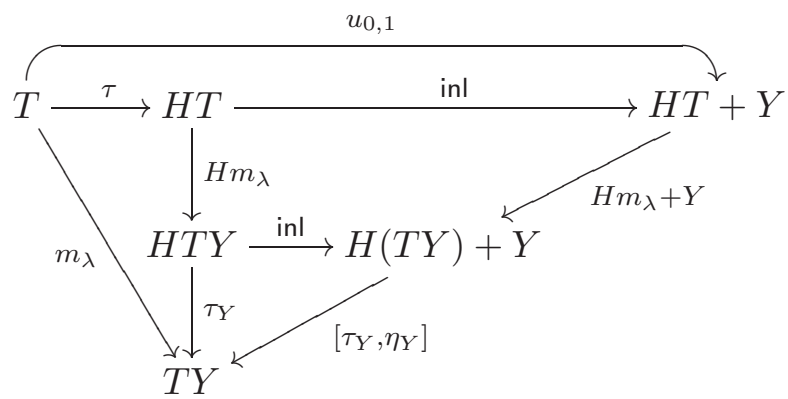

The isolated step is easy because if the compatibility holds for $i$, then the diagram

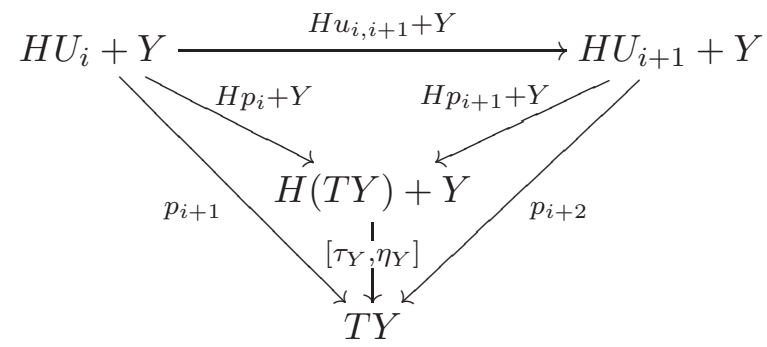

commutes. It is obvious (by transfinite induction) that all $p_{i}$ 's are monomorphisms. It remains to verify that $p_{\lambda}: U_{\lambda} \rightarrow T Y$ is an algebra homomorphism. Indeed, since $H$ preserves $\lambda$-filtered colimits, the corecursive chain $U_{i}$ converges after $\lambda$ steps, so that $U_{\lambda}=$ 
$T \oplus F Y$, by Proposition 4.4. We have following commutative square:

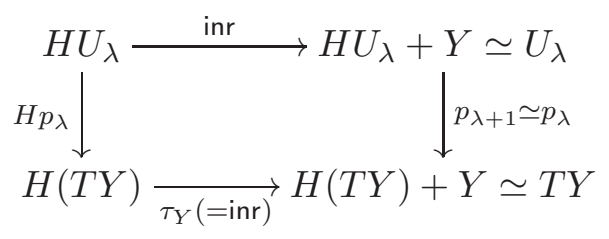

Example 4.7. Free corecursive algebras $M Y$ that are obtained as $U_{\omega}$.

(1) For $H=I d$ we have

$$
M Y=U_{\omega}=1+Y+Y+Y+\ldots
$$

Indeed, the terminal object 1 is $T$, and

$$
U_{1}=T+Y \quad U_{2}=T+Y+Y
$$

with colimit $U_{\omega}=1+Y+Y+Y+\cdots$.

(2) More generally, let $H: \mathcal{A} \rightarrow \mathcal{A}$ preserve countable coproducts and have a terminal coalgebra $T$. Then

$$
M Y=U_{\omega}=T+Y+H Y+H^{2} Y+\cdots
$$

(3) For the endofunctor $H X=X \times X$ of Set (of binary algebras) we have the free corecursive algebra

$M Y=$ all binary trees with finitely many leaves, all of which are labelled in $Y$.

Indeed, the corecursive chain $U$ yields

$$
U_{0}=1
$$

which we represent by the complete binary tree $t$. Then

$$
U_{1}=1 \times 1+Y \simeq 1+Y
$$

is represented by $t$ and all singleton trees labelled in $Y$, and

$$
U_{2}=(1+Y) \times(1+Y)+Y
$$

is represented by all binary trees with leaves of depth at most 1 labelled in $Y$. We conclude that

$$
U_{n}=\text { all binary trees with no leaves of depth } \geq n \text { and leaves labelled in } Y \text {. }
$$

Consequently, the corecursive chain yields

$$
T \oplus F Y=\operatorname{colim}_{n<\omega} U_{n}=\bigcup_{n<\omega} U_{n}
$$

which is the above set of trees. Observe that this description of $M Y$ corresponds well with the fact that $M Y$ is a free binary algebra with an additional idempotent (see Example 2.6): the unique idempotent of $M Y$ is the complete binary tree. And $M Y$ is generated by this tree and all finite trees in $F Y$.

(4) More generally, let $\Sigma=\left(\Sigma_{k}\right)_{k<\omega}$ be a signature. Then $\Sigma$-algebras are precisely the algebras for the polynomial endofunctor

$$
H_{\Sigma} X=\Sigma_{0}+\Sigma_{1} \times X+\Sigma_{2} \times X^{2}+\cdots
$$

Recall that the terminal coalgebra is the coalgebra of all $\Sigma$-trees, that is, trees labelled in $\Sigma$ so that every node with a label of arity $n$ has precisely $n$ children. And $F Y$ is the 
algebra of all finite $(\Sigma+Y)$-trees, where members of $Y$ are considered to have arity 0 . Then $U_{n}$ is the set of all $(\Sigma+Y)$-trees with no leaf of depth greater than $n$ having a label from $Y$. (That is, all leaves on level $n$ or more are labelled by a nullary symbol in $\Sigma_{0}$.) Consequently the free corecursive algebra $\operatorname{colim}_{n<\omega} U_{n}=\bigcup_{n<\omega} U_{n}$ is

$$
\begin{aligned}
M Y=T \oplus F Y= & \text { all }(\Sigma+Y) \text {-trees in which finitely many leaves are la- } \\
& \text { belled in } Y \text { and other leaves labelled in } \Sigma_{0} .
\end{aligned}
$$

(5) For the finite power set functor $\mathcal{P}_{f}$, J. Worrell 34] described the terminal coalgebra $T$ as the coalgebra of all finitely branching, non-ordered, strongly extensional trees. Recall that a (non-ordered) tree is called strongly extensional if for every node, the subtrees rooted at distinct children of the given node are not tree bisimilar. The free corecursive algebra is

$$
\begin{aligned}
T \oplus F Y= & \text { all finitely branching, strongly extensional trees with finitely } \\
& \text { many leaves labelled in } Y \text { (and other leaves unlabelled). }
\end{aligned}
$$

Indeed, this is analogous to (3) since

$$
\begin{aligned}
U_{1} \simeq T+Y= & \text { all the trees in } T \text { and all singleton trees labelled in } Y, \\
U_{2}=\mathcal{P}_{f} U_{1}+Y= & \text { all finitely branching, strongly extensional trees with } \\
& \text { some leaves of depth less than } 2 \text { labelled in } Y,
\end{aligned}
$$$$
\text { etc. }
$$

Thus $T \oplus F Y=\bigcup_{n<\omega} U_{n}$ is the above set of trees. The corecursive chain converges in $\omega$ steps because $\mathcal{P}_{f}$ preserves $\omega$-colimits.

Example 4.8. Now we illustrate the need of $U_{i}$ for infinite ordinals $i$. The functor $H$ : Set $\rightarrow$ Set with $H X=X^{\mathbb{N}}$ has the following free corecursive algebras, where $t_{0}$ is the is the complete countably branching tree:

$$
\begin{aligned}
M Y= & \text { all countably branching trees with leaves labelled in } Y \text { such } \\
& \text { that every infinite path contains a node whose subtree is } t_{0} .
\end{aligned}
$$

To see this, represent $U_{0}=1$ by the single tree $t_{0}$ and obtain

$$
\begin{aligned}
& U_{1} \simeq T+Y \quad=\text { all singleton trees labelled in } Y \text {, plus } t_{0} \\
& U_{2} \simeq(T+Y)^{\mathbb{N}}+Y=\text { all countably branching trees with leaves at levels at most } 1 \text {, } \\
& \text { all of which are labelled in } Y \\
& U_{\omega} \simeq T+Y=\text { all countably branching trees which, for some } n<\omega, \text { have } \\
& \text { all leaves at level at most } n \text {, and they are labelled in } Y \text {. }
\end{aligned}
$$

But $U_{\omega}$ is not an algebra because it does not contain the following tree

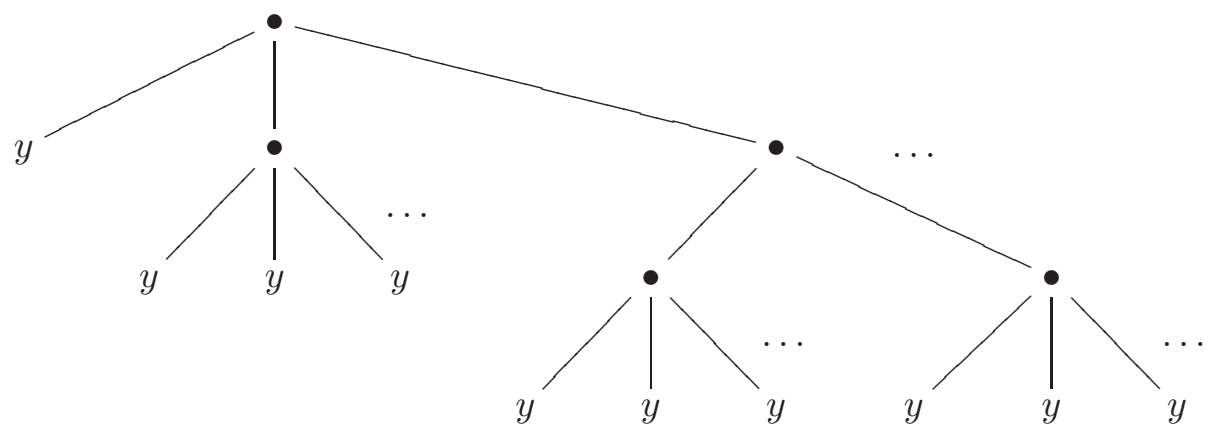


This tree is in $U_{\omega+1}=U_{\omega}^{\mathbb{N}}+Y$.

We can identify, for every ordinal $i$, the set $U_{i}$ with the set of all countably branching trees of type $i$ where type 0 means the tree is $t_{0}$, type $i+1$ means that all maximal subtrees have type $i$, and for limit ordinals $j$, type $j$ means type $i$ for some $i<j$. Then

$$
U_{\omega_{1}}=\text { all countably branching trees of countable type. }
$$

It is easy to verify that a tree has countable type if and only if on every infinite path there exists a node whose subtree is $t_{0}$. Thus, $U_{\omega_{1}}=T \oplus F Y$ is the above free corecursive algebra.

For the proof of Theorem 4.11, the main result of this section, we are going to use the following technical lemma concerning fixpoints and coproducts of algebras.

Lemma 4.9. Let $a: H A \stackrel{\sim}{\rightarrow} A$ be a fixpoint and $B=H B+Y$. If the algebras $(A, a)$ and $(B, \mathrm{inl})$ have a coproduct $(C, c)=(A, a) \oplus(B$, inl) with injections $i$ and $j$ in $\operatorname{Alg} H$, then we have a coproduct $C=H C+Y$ in $\mathcal{A}$ with injections

$$
c: H C \rightarrow C \quad \text { and } \quad d \equiv Y \stackrel{\text { inr }}{\rightarrow} B \stackrel{j}{\rightarrow} C
$$

Proof. For the algebra $\bar{C}=H C+Y$ with structure

$$
\bar{c} \equiv H(H C+Y) \stackrel{H[c, d]}{\longrightarrow} H C \stackrel{\text { inl }}{\longrightarrow} H C+Y
$$

we observe that $a^{-1} \cdot H i \cdot$ inl $: A \rightarrow \bar{C}$ and $H j+i d: B \rightarrow \bar{C}$ are homomorphisms. Indeed, for the former we have the commutative diagram

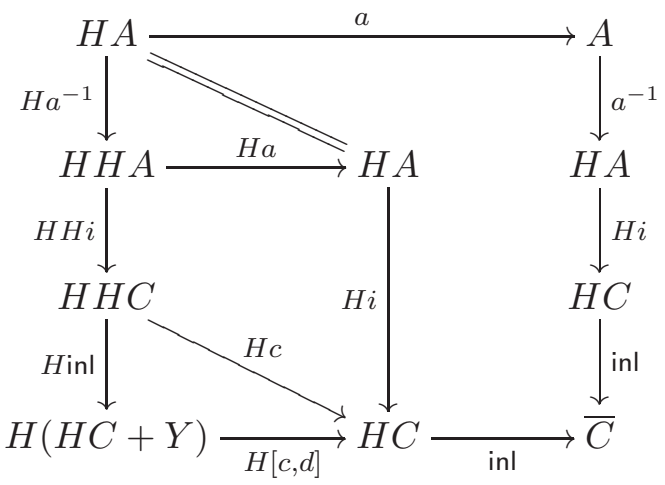

whose middle left-hand part commutes because $i$ is a homomorphism. For the latter one consider the diagram

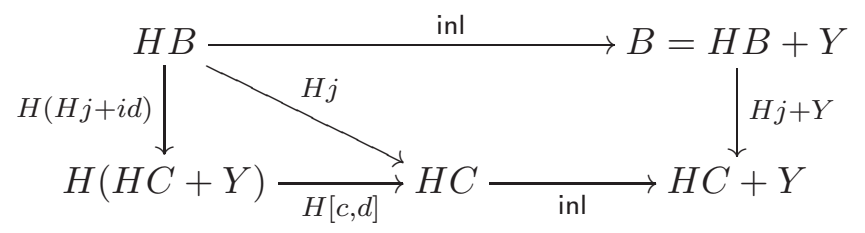

The left-hand triangle commutes because $d=j$.inr and because $j$ is a homomorphism, that is, $c \cdot H j=j \cdot$ inl. Therefore, we have a unique homomorphism $h: C \rightarrow \bar{C}$ with

$$
h \cdot i=\mathrm{inl} \cdot H i \cdot a^{-1}
$$

and

$$
h \cdot j=H j+i d .
$$


This is the inverse of $[c, d]: \bar{C} \rightarrow C$. Indeed, we prove $[c, d] \cdot h=i d$ by using the universal property of coproducts. Firstly, $[c, d]$ is a homomorphism:

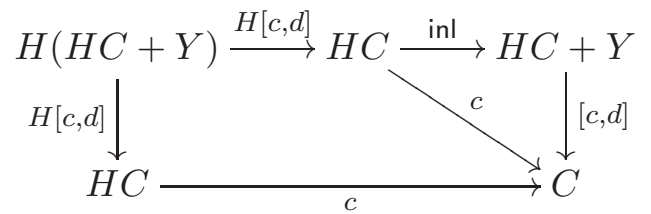

Therefore, $[c, d] \cdot h$ is an endomorphism of $(C, c)$. And we have both

$$
\begin{array}{rlrl}
{[c, d] \cdot h \cdot i} & =[c, d] \cdot \mathrm{inl} \cdot H i \cdot a^{-1} & & \text { by (4.1) } \\
& =c \cdot H i \cdot a^{-1} & & \\
& =i \cdot a \cdot a^{-1} & & i \text { is a homomorphism } \\
& =i &
\end{array}
$$

and

$$
\begin{array}{rlrl}
{[c, d] \cdot h \cdot j} & =[c, d] \cdot(H j+i d) & & \text { by (4.2) } \\
& =[c \cdot H j, d] & \\
& =[j \cdot \text { inl, } j \cdot \text { inr }] & & \text { by (4.1) } \\
& =j . &
\end{array}
$$

The remaining identity $h \cdot[c, d]=i d$ translates into $h \cdot c=$ inl and $h \cdot d=$ inr. The former equality follows from $[c, d] \cdot h=i d$ and the fact that $h$ is a homomorphism

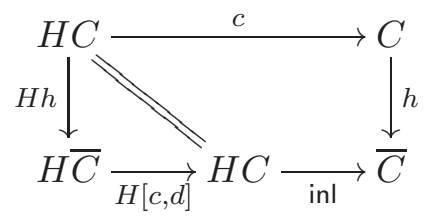

the latter one follows from (4.1) and (4.2) since $h \cdot d=h \cdot j \cdot \mathrm{inr}=(H j+i d) \cdot \mathrm{inr}=\mathrm{inr}$.

\section{Remark 4.10.}

(a) A pre-fixpoint of a functor $H$ is an object $A$ such that $H A$ is a subobject of $A$.

(b) A fixpoint, i. e. an object $A \simeq H A$, can be considered as an algebra or a coalgebra for $H$. When we speak about corecursive fixpoints, we mean fixpoints $\alpha: H A \stackrel{\sim}{\rightarrow} A$ that are corecursive algebras.

Theorem 4.11. For every set functor, the following statements are equivalent:

(i) free corecursive algebras exist,

(ii) free algebras and a terminal coalgebra exist, and

(iii) arbitrarily large pre-fixpoints and a corecursive fixpoint exist.

They imply that the free corecursive algebra on $Y$ is $T \oplus F Y$.

Proof. We can assume, without loss of generality, that $H$ preserves monomorphisms: see the argument for Corollary 3.14. The equivalence of (ii) and (iii) follows from the fact that (a) arbitrarily large pre-fixpoints are necessary and sufficient for the existence of free algebras, see Theorem II.4 in [33] and (b) every corecursive fixpoint is an initial corecursive algebra, thus, a terminal coalgebra, see Proposition 7 in [21]. 
Let us prove (i) $\Leftrightarrow$ (ii).

(i) $\Rightarrow$ (ii): This follows from Lemma 3.9 and Proposition 3.13 which were formulated for Bloom algebras, but hold for corecursive algebras as well. The condition $\operatorname{Set}(Y, H Y) \neq \emptyset$ is automatic for all set functors except the constant functor on $\emptyset$, and (ii) is trivial in that case.

(ii) $\Rightarrow$ (i): Precisely as in the proof of Theorem 4.6 we need to prove that $T \oplus F Y$ exists and is a subalgebra of a corecursive algebra. The existence is clear, since Alg $H$ is cocomplete. Indeed, the existence of free algebras implies that $H$ generates a free monad $\mathbb{F}$, and then Alg $H$ is equivalent to the category $\mathbf{S e t}^{\mathbb{F}}$ of Eilenberg-Moore algebras for $\mathbb{F}$, see [18. And monadic algebras over Set always form cocomplete categories. We prove that the chain from Construction 4.1 converges, thus, from Proposition 4.4 we derive $T \oplus F Y=U_{\gamma}$ for some ordinal $\gamma$. We apply Lemma 4.9 to the coproduct $(C, c)=\left(T, \tau^{-1}\right) \oplus\left(F Y, \phi_{Y}\right)$ with injections inl, inr: put $d=\mathrm{inr} \cdot \eta_{Y}: Y \rightarrow C$, then $C=H C+Y$ with injections $c, d$. Let us define a cocone $m_{i}: U_{i} \rightarrow C$ of the chain $U_{i}$ by $m_{0}=\overline{\mathrm{inl}}: T \rightarrow C$ and

$$
m_{i+1} \equiv H U_{i}+Y \stackrel{H m_{i}+i d}{\longrightarrow} H C+Y \stackrel{[c, d]}{\longrightarrow} C .
$$

We verify that $m_{i}$ form a cocone: the first triangle commutes since $\overline{\text { inl }}$ is a homomorphism:

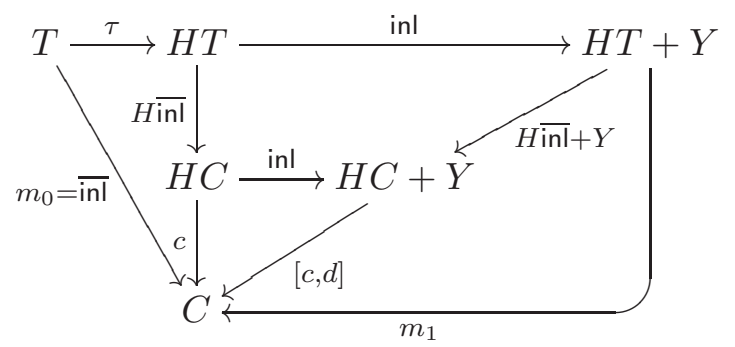

If the $i$-th triangle commutes, it is immediately seen that the next one commutes, too. Furthermore, each $m_{i}$ is a monomorphism. Indeed, $m_{0}=\overline{\mathrm{inl}}$ is a split monomorphism: choose any morphism $f_{0}: Y \rightarrow T$ in Set and extend it to a homomorphism $f:\left(F Y, \phi_{Y}\right) \rightarrow$

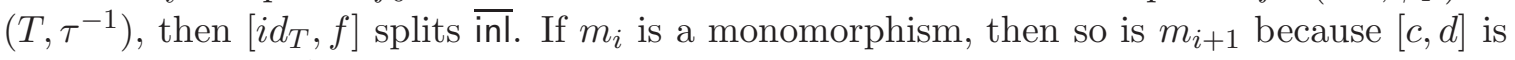
an isomorphism. And limit steps are clear.

Since $C$ has only a set of subobjects, we know that there exist $\delta>\gamma$ such that $m_{\gamma}$ and $m_{\delta}$ represent the same subobject, i. e., $u_{\gamma, \delta}: U_{\gamma} \rightarrow U_{\delta}$ is an isomorphism. Then also $u_{\gamma+1, \delta+1}: U_{\gamma+1} \rightarrow U_{\delta+1}$ is clearly an isomorphism with an inverse $u^{\prime}: U_{\delta+1} \rightarrow U_{\gamma+1}$, say. It follows that $u_{\delta, \delta+1}$ is an isomorphism, too: it is a monomorphism since $m_{\delta+1} \cdot u_{\delta, \delta+1}=m_{\delta}$ and a split epimorphism since $u_{\delta, \delta+1} \cdot u_{\gamma+1, \delta} \cdot u^{\prime}=i d$. Thus we conclude that $T \oplus F Y=U_{\delta}$.

The rest of the proof is analogous to the proof of Theorem 4.6. except that we are not using the terminal coalgebra $T Y$ of $H(-)+Y$ (which need not exist), but one of its "approximations" given by the members $\bar{W}_{i}$ of the terminal chain. I more detail, for every ordinal $i$ the algebra $\bar{w}_{i+1, i}: H \bar{W}_{i}+Y \rightarrow \bar{W}_{i}$ for $H(-)+Y$ is corecursive (apply Example 2.3 (5) for $H(-)+Y)$. It is easy to derive that $\bar{W}_{i}$ is corecursive also when considered as an algebra for $H$ :

$$
H \bar{W}_{i} \stackrel{\text { inr }}{\longrightarrow} H \bar{W}_{i}+Y \stackrel{\bar{w}_{i+1, i}}{\longrightarrow} \overline{W_{i}}
$$

Now the existence of the terminal coalgebra $T$ for $H$ implies, as proved in [6], that the terminal chain $W$ for $H$ converges. Let $\lambda$ be an ordinal with $T=W_{\lambda}$ and $\tau=w_{\lambda+1, \lambda}^{-1}$. As 
in the proof of Theorem 4.6 we define a natural transformation $m_{i}: W_{i} \rightarrow \bar{W}_{i}$ by

$$
m_{0}=i d_{1} \quad \text { and } \quad m_{i+1}=\mathrm{inl} \cdot H m_{i}
$$

and after $\lambda$ steps obtain a homomorphism $m_{\lambda}: T \rightarrow \bar{W}_{\lambda}$ of algebras for $H$ which is monic. And then we obtain a cocone of the corecursive chain $p_{i}: U_{i} \rightarrow \bar{W}_{\lambda}$ by

$$
p_{0}=m_{\lambda} \quad \text { and } \quad p_{i+1} \equiv H U_{i}+Y \stackrel{H p_{i}+i d}{\longrightarrow} H \bar{W}_{\lambda}+Y \stackrel{\bar{w}_{\lambda+1, \lambda}}{\longrightarrow} \bar{W}_{\lambda} .
$$

Analogously to the proof of Theorem 4.6, if the corecursive chain converges after $\gamma$ steps, then

$$
p_{\gamma}: T \oplus F Y=U_{\gamma} \rightarrow \bar{W}_{\lambda}
$$

is the desired monomorphism in Alg $H$.

Notation 4.12. Let $H$ have free corecursive algebras $M Y$. Denote by

$$
\delta_{Y}: H M Y \rightarrow M Y
$$

the algebra structure and by

$$
\eta_{Y}: Y \rightarrow M Y
$$

the universal map. Then we obtain a unique homomorphism

$$
\mu_{Y}:\left(M M Y, \delta_{M Y}\right) \rightarrow\left(M Y, \delta_{Y}\right)
$$

with $\mu_{Y} \cdot \eta_{M Y}=i d$ :

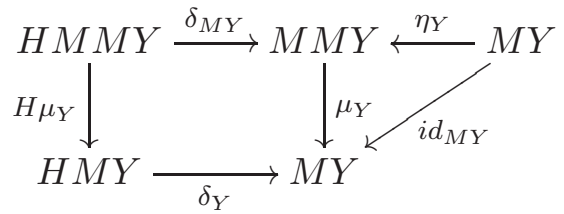

The triple

$$
\mathbb{M}=(M, \mu, \eta)
$$

is the monad generated by the adjoint situation

$$
\operatorname{Alg}_{C} H_{\stackrel{\perp}{\longleftarrow}}^{\longleftarrow} \mathcal{A}
$$

Example 4.13. We have

$$
M Y=\mathbb{N} \times Y+1
$$

for the identity functor on Set, see Example 4.7(1). And for $H X=X \times X$ we have

$M Y=$ binary trees with finitely many leaves, all of which are labelled in $Y$, see Example 4.7(3). The functor $H X=\coprod_{n<\omega} X^{n}$ generates the monad

$M Y=$ finitely branching trees with finitely many leaves labelled in $Y$

cf. Example 4.7(4) for $\Sigma$ with one $n$-ary operation for every $n<\omega$.

Remark 4.14. Since $\mu_{Y}$ is, by definition, a homomorphism:

$$
\mu_{Y} \cdot \delta_{M Y}=\delta_{Y} \cdot H \mu_{Y}
$$

the unit law $\mu_{Y} \cdot \eta_{M Y}=i d$ yields

$$
\delta_{Y}=\mu_{Y} \cdot \delta_{M Y} \cdot H \eta_{M Y} .
$$

It easy to prove that the $\delta_{Y}$ are the components of a natural transformation $\delta: H M \rightarrow M$. 
Theorem 4.15. Let $\mathcal{A}$ be a locally presentable category with constructive monomorphisms, and let $H: \mathcal{A} \rightarrow \mathcal{A}$ be an accessible endofunctor preserving monomorphisms. Then Bloom algebras are precisely the Eilenberg-Moore algebras for $\mathbb{M}$, i.e., the category $\operatorname{Alg}_{B} H$ is isomorphic to $\mathcal{A}^{\mathbb{M}}$.

Proof. The forgetful functors $U_{B}: \operatorname{Alg}_{B} \rightarrow \mathcal{A}$ and $U_{C}: \operatorname{Alg}_{C} H \rightarrow \mathcal{A}$ are both right adjoints, and their left adjoints are, in both cases, defined by $Y \mapsto T \oplus F Y$, see Theorem 3.16 and Proposition 4.4. Therefore the monads generated by $U_{B}$ and $U_{C}$ are the same, namely the $\operatorname{monad} \mathbb{M}$. We are going to prove that the comparison functor from $\operatorname{Alg}_{B} H$ to $\mathcal{A}^{\mathbb{M}}$ is an isomorphism. By Beck's Theorem, see [20, 4.4.4], all we need to prove is that $U_{B}$ creates coequalizers of $U_{B}$-split pairs. That is, we need to prove that given a parallel pair of solution preserving homomorphisms

$$
f, g:(A, a, \dagger) \rightarrow(B, b, \ddagger)
$$

and given morphisms $k, s, t$ in $\mathcal{A}$ as follows:

$$
\begin{array}{ccc}
k: B \rightarrow C & \text { with } & k \cdot f=k \cdot g \\
s: C \rightarrow B & \text { with } & k \cdot s=i d_{A}
\end{array}
$$

and

$$
t: B \rightarrow A \quad \text { with } \quad s \cdot k=f \cdot t \quad \text { and } \quad i d_{B}=g \cdot t
$$

there exists a unique structure $(C, c, *)$ of a Bloom algebra such that $k$ is a solution preserving homomorphism; moreover, $k$ is then a coequalizer in $\operatorname{Alg}_{B} H$. Since $H$ is accessible, free $H$-algebras exist, see Corollary 3.10 . The corresponding monad is the free monad on $H$ and its Eilenberg-Moore algebras are precisely the $H$-algebras, see [18. Consequently, by Beck's Theorem there exists a unique structure $c: H C \rightarrow C$ of an algebra for which $k:(B, b) \rightarrow(C, c)$ is a coequalizer in Alg $H$. And by Lemma 3.7 there exists a unique structure $(C, c, *)$ of a Bloom algebra for which $k$ is a solution preserving algebra homomorphism. It remains to verify that $k$ is a coequalizer in $\mathrm{Alg}_{B} H$. To this end, let

$$
h:(B, b, \ddagger) \rightarrow(D, d,+)
$$

be a solution preserving algebra homomorphism with $h \cdot f=h \cdot g$. There exists a unique homomorphism $h^{\prime}:(C, c) \rightarrow(D, d)$ with $h=h^{\prime} \cdot k$. And $h^{\prime}$ preserves solutions (that is for every $e: X \rightarrow H X$ we have $h^{\prime} \cdot e^{*}=e^{+}$) because both $k$ and $h$ do:

$$
h^{\prime} \cdot e^{*}=h^{\prime} \cdot k \cdot e^{\ddagger}=h \cdot e^{\ddagger}=e^{+} .
$$

Remark 4.16. In the case $\mathcal{A}=$ Set we do not need to assume accessibility: whenever an endofunctor $H$ has free corecursive algebras, then Bloom algebras are precisely the algebras for the monad $\mathbb{M}$. The proof just uses Theorem 4.11 in lieu of 4.4

Example 4.17. A non-accessible set functor having free corecursive algebras. Let $\mathbb{A}$ and $\mathbb{B}$ be proper classes of infinite cardinals such that for every cardinal $\alpha \in \mathbb{A}$ the interval $\left[\alpha, 2^{\alpha}\right]$ is disjoint with $\mathbb{B}$. The functor $H$ assigns to every set $X$ the Set

$$
H X=\{M \subseteq X \mid \text { card } M \in \mathbb{B} \text { or } M=\emptyset\}
$$

and to every function $f: X \rightarrow Y$ the following function

$$
H f(M)= \begin{cases}f[M] & \text { if } f \text { is monic when restricted to } M \\ \emptyset & \text { otherwise }\end{cases}
$$


Then $H$ has the terminal coalgebra 1 (since $H 1=1$ ). It also has free algebras. Indeed, this follows from the fact that $H$ has arbitrary large pre-fixpoints (see the proof of Theorem 4.11): for every cardinal $\alpha \in \mathbb{A}$ the cardinal $2^{\alpha}$ is a pre-fixpoint due to

$$
\text { card } H\left(2^{\alpha}\right) \leq 1+\Sigma_{\beta \in \mathbb{B}, \beta \leq 2^{\alpha}}\left(2^{\alpha}\right)^{\beta} \leq \Sigma_{\beta<\alpha}\left(2^{\alpha}\right)^{\beta} \leq \alpha \cdot\left(2^{\alpha}\right)^{\omega}=2^{\alpha} .
$$

However, $H$ is not $\lambda$-accessible for any $\lambda$ : choose $\beta \in \mathbb{B}$ with $\beta \geq \lambda$, then since $\beta \in H \beta$ we see that $H$ does not preserve the lambda-directed colimit of $\beta$ as the union of all subsets of $\beta$ of cardinality less than $\lambda$.

\section{Corecursive monads}

The iterative theories (or iterative monads) of C. Elgot [22] were introduced as a formalization of iteration in an algebraic setting, and in [23] completely iterative theories were studied. We first recall the concept of a completely iterative monad, and then introduce the weaker concept of a corecursive monad. The relationship between these two concepts is analogous to the relationship between cia's, see Remark 2.7, and corecursive algebras. The following definition is, for the base category Set, equivalent to completely iterative theories, as shown in [1].

Definition 5.1. (See [1])

(1) An ideal monad is a sixtuple

$$
\mathbb{S}=\left(S, \eta, \mu, S^{\prime}, \sigma, \mu^{\prime}\right)
$$

consisting of a monad $(S, \eta, \mu)$, a subfunctor $\sigma: S^{\prime} \rightarrow S$ (called the ideal of $\mathbb{S}$ ) such that $S=S^{\prime}+I d$ with injections $\sigma$ and $\eta$, and a natural transformation $\mu^{\prime}: S^{\prime} S \rightarrow S^{\prime}$ restricting $\mu$, i.e., with $\sigma \cdot \mu^{\prime}=\mu \cdot \sigma S$.

(2) An equation morphism with parameters for $\mathbb{S}$ is a morphism $e: X \rightarrow S(X+Y)$, we call $X$ the variables and $Y$ the parameters of $e$. It is called ideal if it factorizes through $\sigma_{X+Y}$. A solution of $e$ is a morphism $e^{\dagger}: X \rightarrow S Y$ such that the following square commutes:

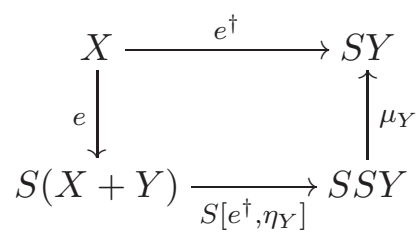

(3) An ideal monad is called completely iterative provided that every ideal equation morphism has a unique solution.

Example 5.2. (See [1]) Let $H$ be an endofunctor of $\mathcal{A}$ such that for every object $Y$ a terminal coalgebra $T Y$ of $H(-)+Y$ exists. Then the assignment $Y \mapsto T Y$ yields a monad $(T, \eta, \mu)$, which is the monad of free cia's for $H$. This is an ideal monad w.r.t. $T^{\prime}=H T$ and $\mu^{\prime}=H \mu$. Moreover, this monad is completely iterative, indeed, the free completely iterative monad on $H$.

For example the set functor $H X=X \times X$ generates the free completely iterative monad $\mathbb{T}$ with

$$
T Y=\text { all binary trees with leaves labelled in } Y .
$$


Definition 5.3. Let $\mathbb{S}$ be an ideal monad. An equation morphism (without parameters) is a morphism $e: X \rightarrow S X$, and $e$ is called ideal if it factorizes through $\sigma_{X}$, i. e., there exist $e_{0}: X \rightarrow S^{\prime} X$ such that the diagram below commutes:

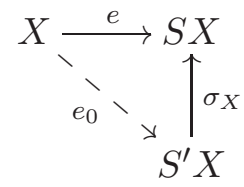

The monad $\mathbb{S}$ is called corecursive if every ideal equation morphism $e$ has a unique solution $e^{\dagger}: X \rightarrow S Y$, i. e., the square below commutes:

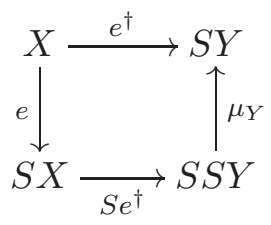

Example 5.4. Examples of corecursive monads on Set.

(1) All the monads of Example 4.7 are corecursive, as we will see in Theorem 6.4 below.

(2) All completely iterative monads are corecursive, e.g,

$$
S Y=\text { all finitely branching trees with leaves labelled in } Y,
$$

This is the free completely iterative monad on the functor $H X=\coprod_{n<\omega} X^{n}$.

(3) Consider the monad

$$
R Y=\text { all rational, finitely branching trees with leaves labelled in } Y,
$$

where rational means that the tree has up to isomorphism only finitely many subtrees. This is a corecursive monad that is neither free on any endofunctor, nor completely iterative.

(4) More generally, every submonad of $S$ in item (2) containing the complete binary tree is corecursive.

Proposition 5.5. The monad $\mathbb{M}=(M, \eta, \mu)$ of free corecursive algebras (of Notation 4.12 ) is ideal w.r.t. the ideal $M^{\prime}=H M$ where $\sigma=\delta: H M \rightarrow M$ and $\mu^{\prime}=H \mu: H M M \rightarrow H M$.

Proof. (1) We prove

$$
M Y=H M Y+Y
$$

with injections $\delta_{Y}$ and $\eta_{Y}$. First apply Lemma 2.5 to the corecursive algebra $M Y$ and $\eta_{Y}: Y \rightarrow M Y$ to see that $H M Y+Y$ is a corecursive algebra, too. Using the freeness of $M Y$ we see that for the right-hand injection morphism inr : $Y \rightarrow H M Y+Y$ there exists a unique homomorphism inr $: M Y \rightarrow H M Y+Y$ such that inr $\cdot \eta_{Y}=$ inr. In order to prove that $\overline{i n r}$ is an inverse for $\left[\delta_{Y}, \eta_{Y}\right]$ we consider the following diagram

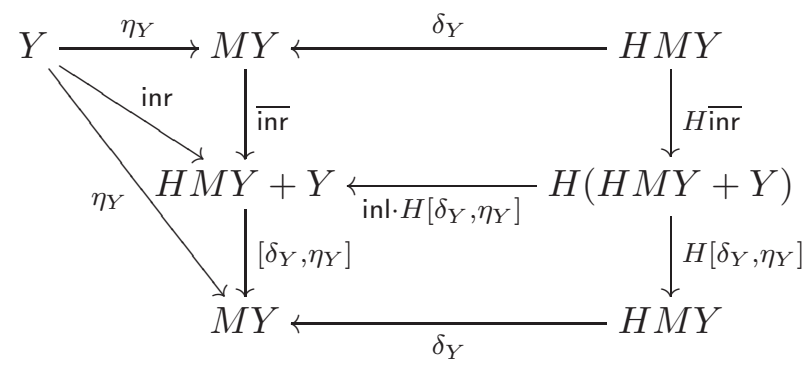


The upper square and triangle commute by the definition of inr, and the lower triangle and square obviously do. Thus $\left[\delta_{Y}, \eta_{Y}\right]$ is homomorphism, and the composition of the two squares clearly commutes. So $\left[\delta_{Y}, \eta_{Y}\right] \cdot \overline{\mathrm{inr}}=i d_{M Y}$ follows from the universal property of $\eta_{Y}$. Now the upper square of (5.2) yields $\overline{\mathrm{inr}} \cdot \delta_{Y}=\mathrm{inl}$, consequently $\left(\overline{\mathrm{inr}} \cdot\left[\delta_{Y}, \eta_{Y}\right]\right) \cdot \mathrm{inl}=$ $\overline{\mathrm{inr}} \cdot \delta_{Y}=\mathrm{inl}$. Since also $\left(\overline{\mathrm{inr}} \cdot\left[\delta_{Y}, \eta_{Y}\right]\right) \cdot \mathrm{inr}=\overline{\mathrm{inr}} \cdot \eta_{Y}=\mathrm{inr}$, we conclude $\overline{\mathrm{inr}} \cdot\left[\delta_{Y}, \eta_{Y}\right]=i d$.

(2) It remains to prove $\sigma \cdot \mu^{\prime}=\mu \cdot \sigma S$, that is $\delta \cdot H \mu=\mu \cdot \delta M$, which one obtains from Diagram (4.3).

Theorem 5.6. The monad $\mathbb{M}$ of free corecursive algebras is corecursive.

Proof. We need to prove that every ideal equation morphism $e=\delta_{X} \cdot e_{0}$ where $e_{0}: X \rightarrow$ $H M X$ has a unique solution in all algebras $\left(M Y, \mu_{Y}\right)$. We prove a stronger statement: if $a: H A \rightarrow A$ is a corecursive algebra, then $e$ has a unique solution in $A$. That means that there exists a unique morphism $e^{\dagger}: X \rightarrow A$ such that the square

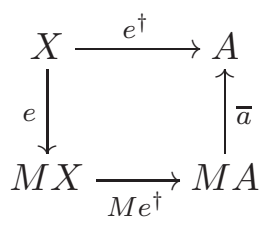

commutes, where $\bar{a}$ is the corresponding Eilenberg-Moore algebra structure (the unique homomorphism of $H$-algebras from $M A$ to $A$ such that $\bar{a} \cdot \eta_{A}=i d_{A}$ ). Form the equation morphism

$$
\bar{e} \equiv M X \stackrel{\left[\delta_{X}, \eta_{X}\right]^{-1}}{\longrightarrow} H M X+X \stackrel{\left[H M X, e_{0}\right]}{\longrightarrow} H M X .
$$

There is a unique solution $s$ of $\bar{e}$ :

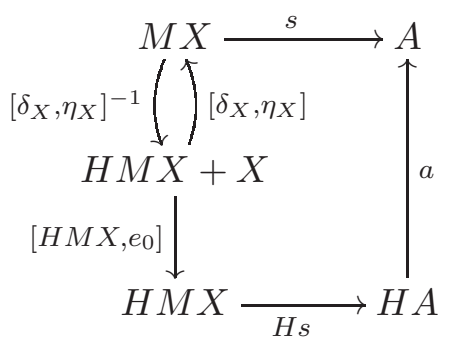

Inspecting the two coproduct components of $H M X+X$ separately, we see that the commutativity of (5.3) is equivalent to the following two equations:

$$
\begin{aligned}
& s \cdot \delta_{X}=a \cdot H s \\
& s \cdot \eta_{X}=a \cdot H s \cdot e_{0}
\end{aligned}
$$

Equation (5.4) states that $s$ is a homomorphism. Also since $\eta: I d \rightarrow M$ is a natural transformation we have the commutative diagram 


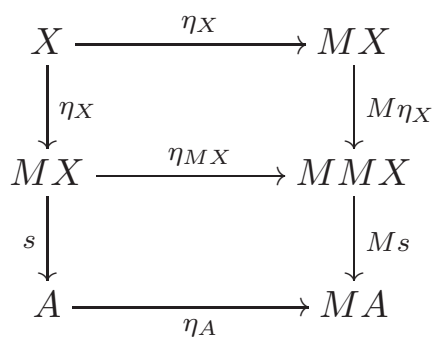

which gives us $\bar{a} \cdot M s \cdot M \eta_{X} \cdot \eta_{X}=\bar{a} \cdot \eta_{A} \cdot s \cdot \eta_{X}=s \cdot \eta_{X}$. From this the universal property of $\eta_{X}$ implies the equation

$$
\bar{a} \cdot M s \cdot M \eta_{X}=s .
$$

We prove that $e^{\dagger}=s \cdot \eta_{X}$ is a solution of $e$ by inspecting the diagram

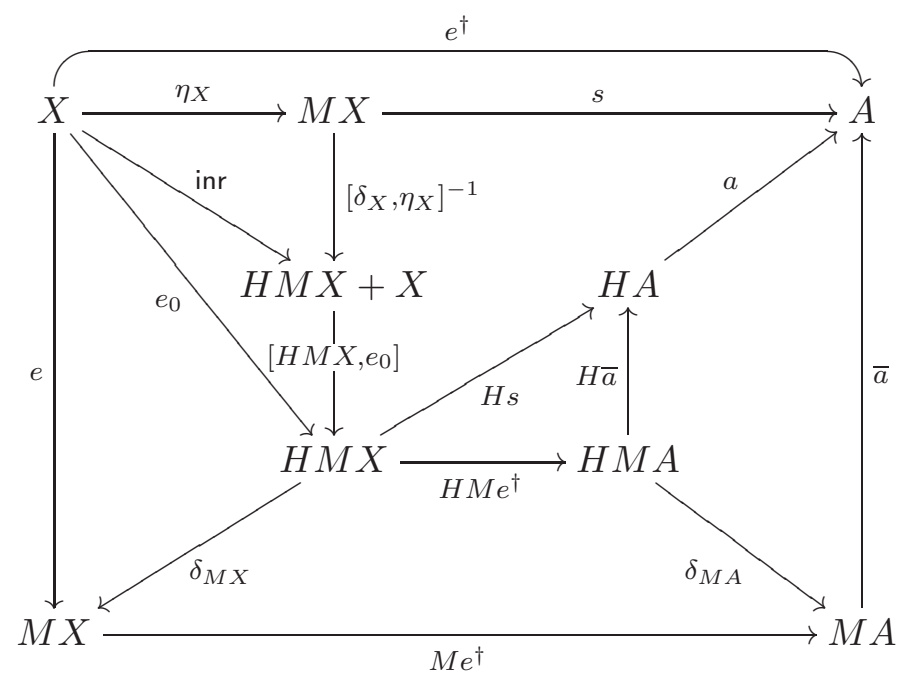

The left-hand triangle commutes because $e$ is an ideal equation. The upper right-hand triangle is Diagram (5.3), the middle triangle commutes because of Equation (5.6) and $e^{\dagger}=s \cdot \eta_{X}$, and the other parts commute obviously.

To show uniqueness, suppose that $e^{\dagger}$ is an arbitrary solution of $e$. We take $s=\bar{a}$. $M e^{\dagger}: M X \rightarrow A$, and prove that $s$ is a solution of $\bar{e}$. First we note that $s$ is an algebra homomorphism for $H$, since both $\bar{a}$ and $M e^{\dagger}$ are, thus Equation (5.4) holds for $s$. We also have $s \cdot \eta_{X}=e^{\dagger}$ using the naturality of $\eta$ :

$$
s \cdot \eta_{X}=\bar{a} \cdot M e^{\dagger} \cdot \eta_{X}=\bar{a} \cdot \eta_{A} \cdot e^{\dagger}=e^{\dagger} .
$$

Now all the parts in Diagram (5.7) commute except, possibly, the upper right-hand triangle. So this triangle commutes when precomposed with $\eta_{X}: X \rightarrow M X$ from which we conclude Equation (5.5). Combining (5.4) and (5.5) we have, equivalently, that Diagram (5.3) commutes, i. e., $s$ is a solution of $\bar{e}$. 


\section{Free Corecursive Monad}

In this section we prove that the corecursive monad $\mathbb{M}$ given by the free corecursive algebras for $H$ is a free corecursive monad on $H$. For that we need the appropriate concept of morphism:

\section{Definition 6.1.}

(a) An ideal monad morphism from an ideal monad $\left(S, \eta^{S}, \mu^{S}, S^{\prime}, \sigma, \mu^{\prime S}\right)$ to an ideal monad $\left(U, \eta^{U}, \mu^{U}, U^{\prime}, \omega, \mu^{\prime U}\right)$ is a pair consisting of a monad morphism $\lambda:\left(S, \eta^{S}, \mu^{S}\right) \rightarrow$ $\left(U, \eta^{U}, \mu^{U}\right)$ and a natural transformation $\lambda^{\prime}: S^{\prime} \rightarrow U^{\prime}$ with $\lambda \cdot \sigma=\omega \cdot \lambda^{\prime}$.

(b) Given a functor $H$, a natural transformation $\lambda: H \rightarrow S$ is called ideal if it factors through $\sigma: S^{\prime} \rightarrow S$.

(c) By a free corecursive monad on an endofunctor $H$ is meant a corecursive monad $\mathbb{S}=$ $\left(S, \mu, \eta, S^{\prime}, \sigma, \mu^{\prime}\right)$ together with an ideal natural transformation $\kappa: H \rightarrow S$ with the following universal property: For every ideal natural transformation $\lambda: H \rightarrow \overline{\mathbb{S}}$, where $\overline{\mathbb{S}}$ is a corecursive monad, there exists a unique ideal monad morphism $\left(\hat{\lambda}, \hat{\lambda}^{\prime}\right): \mathbb{S} \rightarrow \overline{\mathbb{S}}$ such that the triangle below commutes:

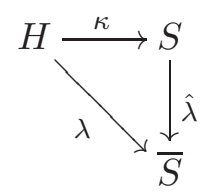

Remark 6.2. Let $\mathrm{CMon}(\mathcal{A})$ denote the category of corecursive monads and ideal monad morphisms. We have a forgetful functor to $\operatorname{Fun}(\mathcal{A}, \mathcal{A})$, the category of all endofunctors of $\mathcal{A}$, assigning to every corecursive monad $\mathbb{S}$ its ideal $S^{\prime}$. A free corecursive monad on $H \in \operatorname{Fun}(\mathcal{A}, \mathcal{A})$ is precisely a universal arrow from $H$ to the above forgetful functor.

Example 6.3. If $H$ has free corecursive algebras, then we have the corecursive monad $\mathbb{M}$ of Proposition 5.5. And the natural transformation

$$
\kappa \equiv H \stackrel{H \eta}{\longrightarrow} H M \stackrel{\delta}{\longrightarrow} M
$$

(see Notation 4.12) is obviously ideal. We prove that $\kappa$ has the universal property:

Theorem 6.4. If an endofunctor $H$ has free corecursive algebras, then the corresponding monad $\mathbb{M}$ is the free corecursive monad on $H$.

Remark 6.5. The proof is analogous to the corresponding theorem for free completely iterative monads, see [28, Theorem 4.3].

Proof. For every corecursive monad

$$
\mathbb{S}=\left(S, \mu^{S}, \eta^{S}, S^{\prime}, \sigma, \mu^{\prime S}\right)
$$

and every ideal natural transformation

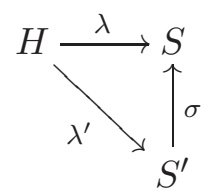

we are going to find an ideal monad morphism $\left(\hat{\lambda}, \hat{\lambda}^{\prime}\right): \mathbb{M} \rightarrow \mathbb{S}$ with $\lambda=\hat{\lambda} \cdot \kappa$, and prove that it is unique. 
(a) Every object $S A, A \in \mathcal{A}$, is considered as an algebra for $H$ via

$$
\rho_{A} \equiv H S A \stackrel{\lambda_{S A}}{\longrightarrow} S S A \stackrel{\mu_{A}^{S}}{\longrightarrow} S A .
$$

We prove that $S A$ is corecursive. Every equation morphism $e: X \rightarrow H X$ yields the following equation morphism $\bar{e} \equiv X \stackrel{e}{\rightarrow} H X \stackrel{\lambda_{X}}{\rightarrow} S X$ w.r.t. the monad $\mathbb{S}$ and, and since $\lambda$ is an ideal natural transformation we see that $e$ an ideal equation morphism. We now prove that $e^{\dagger}$ is a solution of $e$ in $S A$ if and only if it is the solution of $\bar{e}$ with respect to the corecursive monad $\mathbb{S}$. Let $e^{\dagger}$ be a solution of $e$ in $S A$, that is, the upper part of the diagram below commutes:

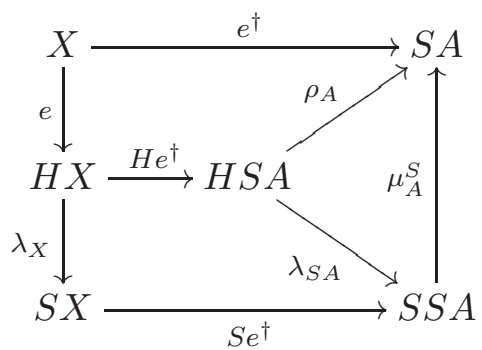

Since the lower part commutes because of naturality of $\lambda$ and the right-hand triangle by definition of $\rho$, we see that $e^{\dagger}$ is a solution of $\bar{e}$.

Conversely, if $e^{\dagger}$ is a solution of $\bar{e}$, then the outside of the above diagram commutes. Consequently, the upper part is commutative, showing $e^{\dagger}$ to be a solution of $e$ as desired. Thus, $S A$ is a corecursive algebra.

(b) There exists a unique homomorphism $\hat{\lambda}_{A}: M A \rightarrow S A$ of $H$-algebras such that $\hat{\lambda}_{A}$. $\eta_{A}=\eta_{A}^{S}$. Now we show that $\hat{\lambda}$ is a natural transformation. Consider $f: A \rightarrow B$, then $S f: S A \rightarrow S B$ is a homomorphism:

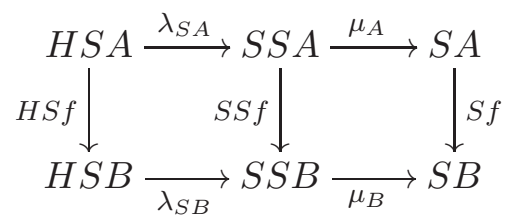

The outside of the following diagram

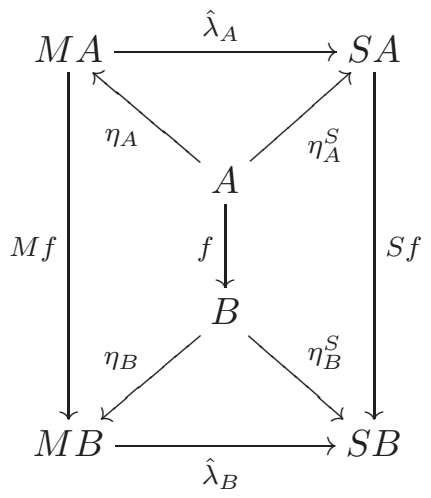

commutes by the universal property of $\eta_{A}$. 
(c) We prove next that $\hat{\lambda}$ is a monad morphism. That is, the following diagrams are commutative:
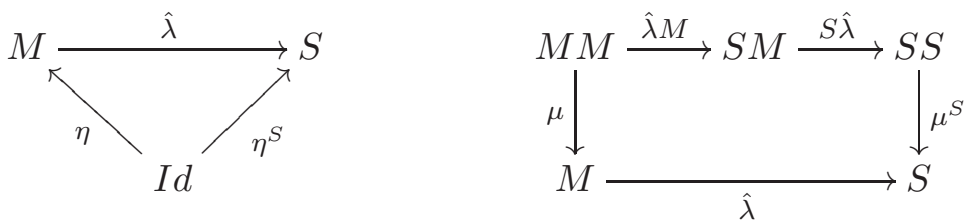

The left-hand triangle commutes because of the definition of $\hat{\lambda}$. For the right hand square we note that $S \hat{\lambda}_{A}$ is a homomorphism (cf. (6.2) with $f=\hat{\lambda}_{A}$ ), all (components of) the other natural transformations are also clearly homomorphisms, and we have the following diagram

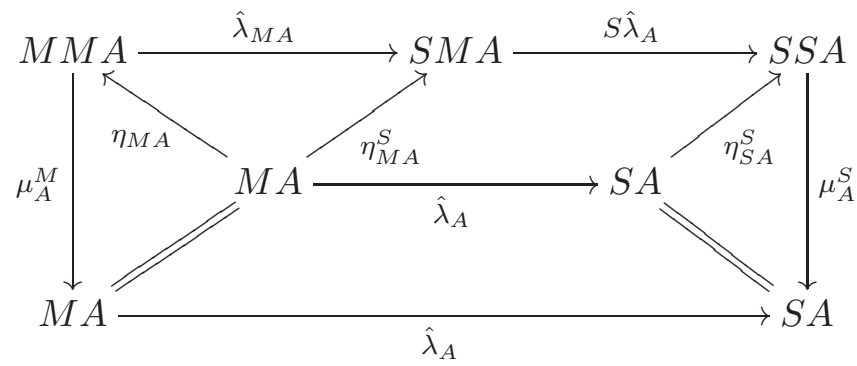

The right-hand square commutes by naturality of $\eta^{S}$, hence the outside square is commutative.

(d) Now we have to show that $\lambda=\hat{\lambda} \cdot \kappa=\hat{\lambda} \cdot \delta \cdot H \eta$ which follows from the commutativity of the diagram below:

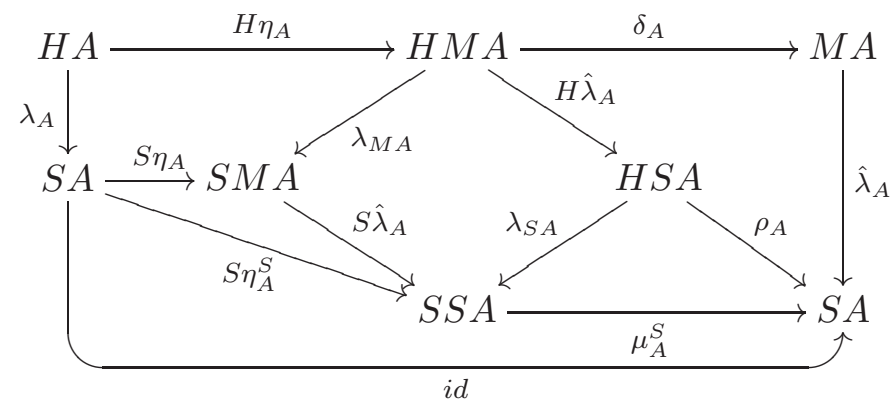

The left-hand upper part and the central one commute because $\lambda$ is an ideal natural transformation. The right-hand upper part commutes by the definition of $\hat{\lambda}$. The lower right-hand triangle commutes by the definition of $\rho$, see (6.1) and the lowest part commutes by the monad laws of $\mathbb{S}$. 
(e) Next we show that $\left(\hat{\lambda}, \hat{\lambda}^{\prime}\right)$, where $\hat{\lambda}^{\prime}=\mu^{\prime S} \cdot \lambda^{\prime} S \cdot H \hat{\lambda}$, is an ideal monad morphism. This follows from

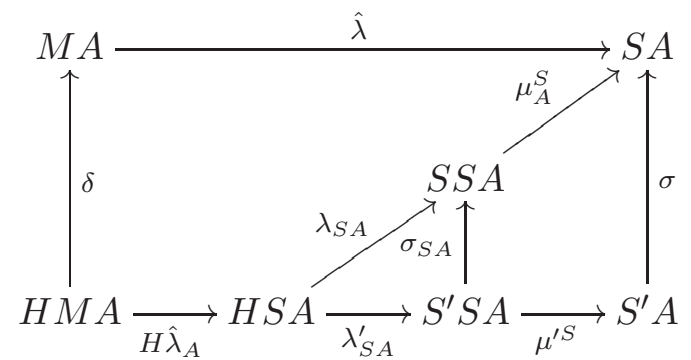

(f) It remains to prove that $\left(\hat{\lambda}, \hat{\lambda}^{\prime}\right)$ is unique. Let $\left(\varphi, \varphi^{\prime}\right): \mathbb{M} \rightarrow \mathbb{S}$ be an ideal monad morphism with $\varphi \cdot \kappa=\lambda$. It is sufficient to prove that $\varphi_{A}: M A \rightarrow S A$ is a homomorphism of $H$-algebras w.r.t. the structure $\rho$ above and $\varphi_{A} \cdot \eta_{A}=\eta_{A}^{S}$, then $\varphi_{A}=\hat{\lambda}_{A}$ of (b) above. From that we derive $\varphi^{\prime}=\hat{\lambda}^{\prime}$ since $\sigma$ is a monomorphism:

$$
\sigma \cdot \varphi^{\prime}=\varphi \cdot \delta=\hat{\lambda} \cdot \delta=\sigma \cdot \hat{\lambda}^{\prime}
$$

The equation $\varphi_{A} \cdot \eta_{A}=\eta_{A}^{S}$ follows from $\varphi$ preserving the units of the monad. And the fact that $\varphi_{A}$ is a homomorphism follows from the following diagram:

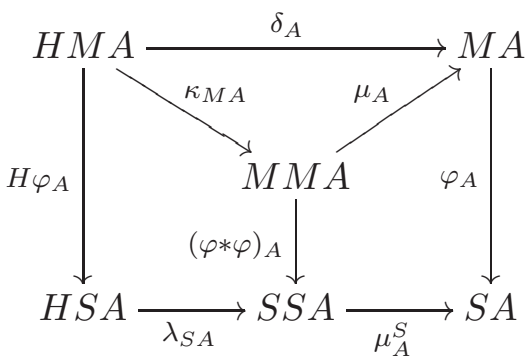

For the upper triangle see Remark 4.14 (and recall that $\kappa=\delta \cdot H \eta$ ), the right-hand square is the preservation of the monad multiplication, and for the left-hand one we use $(\varphi \cdot \kappa) M=$ $\lambda M$ and the naturality of $\lambda$ :

\section{Example 6.6.}

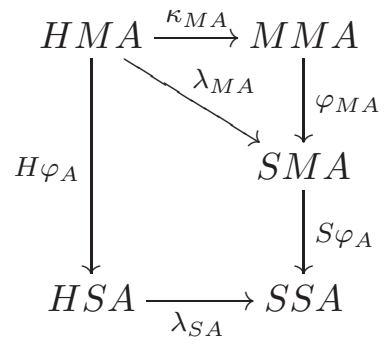

(1) The functor $I d$ generates the free corecursive monad

$$
M Y=\mathbb{N} \times Y+1,
$$

see Example 4.7(1). This is also the free completely iterative monad, since the functor $I d+Y$ has the terminal coalgebra $\mathbb{N} \times Y+1$. 
(2) The polynomial functor $H_{\Sigma}$ of a signature $\Sigma=\left(\Sigma_{n}\right)_{n<\omega}$ generates the free corecursive monad

$M Y=$ all $(\Sigma+Y)$-trees in which only finitely many leaves are labelled in $Y$ (and other leaves labelled in $\Sigma_{0}$ ).

See Example 4.7(4).

Are there any other free corecursive monads than the monads $\mathbb{M}$ of free corecursive algebras? Not for endofunctors of Set:

Proposition 6.7. If a set functor generates a free corecursive monad, then it has free corecursive algebras.

Proof. Let $H:$ Set $\rightarrow$ Set generate a free corecursive monad $\mathbb{S}=\left(S, \mu^{S}, \eta^{S}, S^{\prime}, \sigma, \mu^{\prime}\right)$, and let $\kappa: H \rightarrow S$ be the universal arrow. Following Theorem 4.11 we need to prove the existence of (a) arbitrary large pre-fixpoints and (b) a corecursive fixpoint.

The main technical statement is that the ideal $S^{\prime}$ is naturally isomorphic to $H S$. This proof is analogous to the same proof concerning free completely iterative monads, see Sections 5 and 6 in [1]. We therefore omit it.

Ad (a). Since $S Y=S^{\prime} Y+Y=H S Y+Y$ for every set $Y$, we see that $S Y$ is a pre-fixpoint of cardinality at least card $Y$.

Ad (b). The isomorphism $\sigma_{\emptyset}: H S \emptyset \rightarrow S \emptyset$ defines a corecursive algebra for $H$. To prove this, consider an arbitrary equation morphism $e: X \rightarrow H X$ and form the equation morphism $\bar{e}=\kappa_{X} \cdot e: X \rightarrow S X$. Then solutions of $\bar{e}$ w.r.t $\mathbb{S}$ (in $S \emptyset$ ) are in bijective correspondence with solutions $e$ in the algebra $S \emptyset$. This is easy to prove, the details are as in the of proof of Theorem 6.1 of [28].

\section{Hyper-Extensive Categories}

Recall that for the more general case of recursion with parameters the equational properties of $\dagger$ are captured by iteration theories of S. Bloom and Z. Ésik [19]. Recently functoriality was "added" to these properties; functoriality states that for two equation morphisms with parameters $e: X \rightarrow S(X+Y)$ and $f: Z \rightarrow S(Z+Y)$ (cf. Definition 5.1) we have

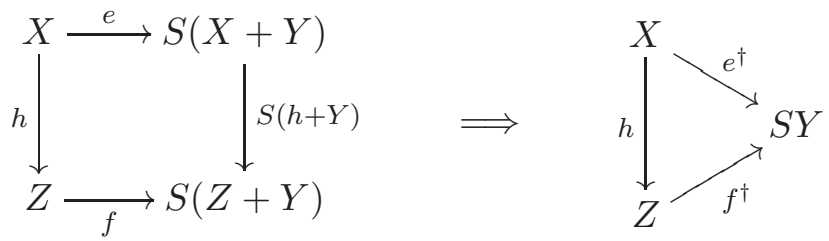

Being an implication, this is not equational if one takes, as in [19], the category of signatures as the base category. Instead, in [12] the presheaf category

$$
\text { Set }^{\mathbb{F}} \text { (sets in context) }
$$

where $\mathbb{F}$ is the category of finite sets and functions, was suggested as a base category. Equivalently, this is the category of all finitary endofunctors on Set. Then functoriality is an equational property in the sense of Kelly and Power [26], and the functorial iteration theories are called Elgot Theories in [12. It follows from the results in [19] that all equational properties of $\dagger$ in Domain Theory are precisely captured by the concept of iteration theory. More precisely, every equation that holds for a parametrized fixpoint operator $\dagger$ given by 
least fixpoints in a category of domains follows from the axioms of iteration theories (see e.g. Simpson and Plotkin [30]).

We have proved in [12] that Elgot theories are monadic over sets in context:

Theorem 7.1 ([12]). Form the monad $\mathcal{M}$ on Set $^{\mathbb{F}}$ by assigning to every set in context $H$ the free iterative theory on $H_{\perp}=H(-)+1$ of $C$. Elgot [22]. Then the Eilenberg-Moore algebras for $\mathcal{M}$ are precisely the Elgot theories.

This result implies, using the results of Kelly and Power [26, that Elgot theories are equational over sets in context, and we gave one axiomatization (that includes functoriality) in 12 .

Example 7.2. The polynomial set functor $H_{\Sigma}$ of Example 4.7(4) defines a set in context (that we also denote by $H_{\Sigma}$ ) by a domain restriction to $\mathbb{F}$. The corresponding iterative theory $\mathcal{M}\left(H_{\Sigma}\right)$ is given by

$$
X \mapsto \text { all rational trees labelled in } \Sigma+X+\{\perp\} .
$$

This is a subtheory of the theory $\mathcal{T}_{\Sigma}$ of all trees labelled in $\Sigma+X+\{\perp\}$, which is the free continuous theory (see Example 3.2(c)).

It was proved by Bloom and Ésik in [19] that the equational properties of the operation $\dagger$ (of solving recursive equations) of the above theory $\mathcal{T}_{\Sigma}$ are precisely the equational properties that $\dagger$ has in an impressive number of applications of iteration. Thus, the axiomatization of these properties in [19] can be understood as the summary of equational properties that $\dagger$ is expected to have in applications.

For every finitary set functor $H$ there exists a signature $\Sigma$ such that $H$ is a quotient of $H_{\Sigma}$ (see [15]). Therefore, $\mathcal{M}(H)$ is a quotient theory of the theory $\mathcal{M}\left(H_{\Sigma}\right)$ of rational trees. Thus, the equational properties of $\dagger$ in all free Elgot theories $\mathcal{M}(H)$ for finitary set functors $H$ are determined by those of $\dagger$ in rational trees.

In the present section we provide the first steps to an analogous result for iteration without parameters. We introduce finitary corecursive monads as the analogy of Elgot's iterative theories, and we prove that every finitary endofunctor on Set generates a free finitary corecursive monad. Let $\mathcal{M}^{*}$ be the monad on Set $^{\mathbb{F}}$ given by

$$
\mathcal{M}^{*}(H)=\text { free finitary corecursive monad on } H_{\perp} \text {. }
$$

Then we prove that the Eilenberg-Moore algebras for $\mathcal{M}^{*}$ are precisely the Bloom theories, i. e., theories with an operation $\dagger$ satisfying the equational properties that hold in nonparametric iteration. We list some of these properties. It is an open problem whether our list is complete.

In lieu of Set we work, more generally, in a locally finitely presentable category. Thus in lieu of theories we work with finitary monads (in analogy to iterative monads of [22]). For most of the results we need to assume the category we work with is hyper-extensive. We now start by recalling this concept from [3].

Definition 7.3. (See [3]) A locally finitely presentable category $\mathcal{A}$ is called hyper-extensive if every object is a coproduct of connected objects, i. e., objects $A$ such that $\mathcal{A}(A,-)$ preserves coproducts.

Remark 7.4. In [3] the definition is different, but Theorem 2.7 of [3] states that the present formulation is equivalent. Every hyper-extensive category is extensive, i. e., coproducts are (a) disjoint (coproduct injections are monic and pairwise intersections always yield 0) and 
(b) universal (preserved by pullback along any morphism).

Moreover, in hyper-extensive categories we have

(c) given pairwise disjoint monics $a_{i}: A_{i} \rightarrow B, i \in \mathbb{N}$, if each $a_{i}$ is coproduct injection then so is $\left[a_{i}\right]: \coprod_{i \in \mathbb{N}} A_{i} \rightarrow B$.

For locally finitely presentable categories (a)-(c) are equivalent to hyper-extensivity.

Example 7.5. Sets, posets, graphs, and every presheaf category are hyper-extensive. Given a signature $\Sigma$ the category of $\Sigma$-algebras is hyper-extensive iff all arities are 1 .

Remark 7.6. In a hyper-extensive category a monad $\mathbb{S}=(S, \mu, \eta)$ is ideal (see Definition 5.1) iff that $S$ is a coproduct $S=S^{\prime}+I d$ with injections $\sigma: S^{\prime} \rightarrow S$ and $\eta: I d \rightarrow S$ and the multiplication has a restriction

$$
\mu^{\prime}: S^{\prime} S \rightarrow S^{\prime}
$$

Thus, in this setting "ideal" is a property not an additional structure of a monad.

Notation 7.7. $\operatorname{Mon}_{i}(\mathcal{A})$ denotes the category of ideal monads and ideal monad morphisms, i.e., morphisms $\alpha: \mathbb{S} \rightarrow \mathbb{T}$ for which a restriction to the ideals exist: we have

$$
\alpha=\left(S=S^{\prime}+I d \stackrel{\alpha^{\prime}+I d}{\longrightarrow} T^{\prime}+I d=T\right)
$$

for a natural transformation $\alpha^{\prime}: S^{\prime} \rightarrow T^{\prime}$.

Remark 7.8. We shall prove below that every corecursive monad $\mathbb{S}$ on a hyper-extensive category has solutions for all, not only ideal, equation morphisms. For that we need to specify an element of $S 0$ which then serves for defining solutions of non-ideal equations such as $x=x$. In the following definition 1 denotes the terminal object of $\mathcal{A}$ and 0 the initial one. The unique morphism from 0 to $X$ is denoted by ! : $0 \rightarrow X$. Analogously $!: X \rightarrow 1$.

Definition 7.9. A strict endofunctor is an endofunctor $H$ together with a morphism $\perp$ : $1 \rightarrow H 0$. A monad is strict if its underlying endofunctor is. A natural transformation $\alpha: H \rightarrow K$ between strict functors is called strict if $\alpha_{0}$ preserves $\perp$.

Every strict endofunctor has a special global element in every $H X$ : it is the composite of $\perp: 1 \rightarrow H 0$ and $H$ ! : H0 $\rightarrow H X$. We denote it again by $\perp: 1 \rightarrow H X$.

Remark 7.10. We now recall from [3] the concept of a strict solution and the fact that every equation morphism has a unique strict solution. In [3] equation morphisms with parameters $Y$ (see Definition [5.1) were considered, here we restrict ourselves to $Y=0$.

Definition 7.11. (See [3]) (a) For every equation morphism $e: X \rightarrow S X$ we denote by

$$
i_{\infty}: X_{\infty} \rightarrow X
$$

the intersection of the derived subobjects $i_{1}, i_{1} \cdot i_{2}, i_{1} \cdot i_{2} \cdot i_{3}, \cdots$ obtained by forming recursively pullbacks as follows:

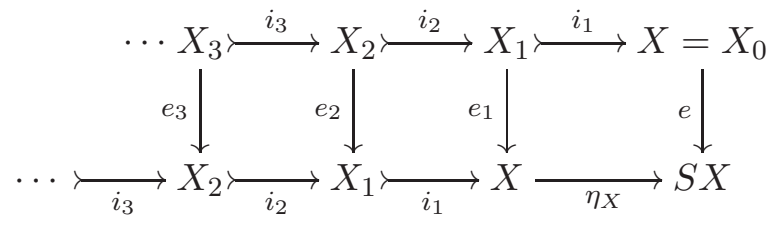


Remark 7.12. $X_{\infty}$ represents those variables for which solutions of $e$ have "difficulties" assigning a value. For example, if $e$ represents the iterative equation $x=x$ or the system

$$
x=y, \quad y=x
$$

then $X=X_{\infty}$. We resolve the difficulties by assigning the value $\perp$ to such variables:

Definition 7.13. (See [3]) Let $e: X \rightarrow S X$ be an equation morphism. A solution $e^{\dagger}: X \rightarrow$ $S Y$ is called strict if its restriction to $X_{\infty}$ factorizes through $\perp$ :

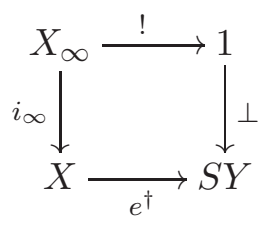

Theorem 7.14. (See [3]) Let $\mathbb{S}$ be a strict, corecursive monad on a hyper-extensive category. Then every equation morphism $e: X \rightarrow S X$ has a unique strict solution $e^{\dagger}: X \rightarrow S Y$, for every object $Y$.

In [3] we proved this for completely iterative monads, the proof for the corecursive monads is the same.

\section{Finitary Bloom algebras and monads}

In this section we investigate the variant of iteration in which only equation morphisms $e: X \rightarrow S X$ with finitely presentable objects $X$ (of variables) are considered.

Throughout this section we assume that the base category is locally finitely presentable and hyper-extensive. And a finitary endofunctor $H$ is given.

\section{Definition 8.1.}

(a) An algebra $a: H A \rightarrow A$ is said to be finitary corecursive if for every coalgebra $e$ : $X \rightarrow H X$ with $X$ finitely presentable there exists a unique solution, i.e., a unique coalgebra-to-algebra morphism $e^{\dagger}: X \rightarrow A$.

(b) A finitary Bloom algebra is a triple $(A, a, \dagger)$ where $a: H A \rightarrow A$ is an algebra and $\dagger$ an operation which to every $e: X \rightarrow H X, X$ finitely presentable, assigns a solution $e^{\dagger}: X \rightarrow A$ subject to functoriality: for every coalgebra homomorphism $h:(X, e) \rightarrow$ $\left(X^{\prime}, e^{\prime}\right)$ with $X$ and $X^{\prime}$ finitely presentable the following triangle commutes:

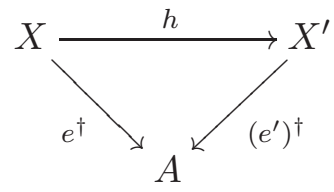

(c) Homomorphisms are defined analogously to Definition 3.3 .

\section{Remark 8.2.}

(a) Every finitary corecursive algebra is a finitary Bloom algebra: the functoriality follows from the uniqueness of solutions.

(b) Lemmas 2.9 and 3.7 hold also for finitary Bloom algebras.

Example 8.3. Consider unary algebras in Set, that is, $H=I d$. 
(a) An algebra $a: A \rightarrow A$ is finitary corecursive iff $a$ has a unique fixpoint $t=a(t)$. Indeed, for every equation morphism $e: X \rightarrow X$ with $X$ finite the unique solution is $e^{\dagger}=$ const $_{t}$.

Thus the algebra $\mathbb{Z}^{+}$of integers with $\infty$ where the unary operation is successor (and $\infty$ is its fixpoint) is finitary corecursive. But not corecursive: consider the system of equations given by $x_{i}=a\left(x_{i+1}\right)$ for $i \in \mathbb{N}$. It has more that one solution in $\mathbb{Z}^{+}$, e.g., $x_{i} \mapsto-i$ and $x_{i} \mapsto \infty$ are solutions.

(b) An algebra $a: A \rightarrow A$ is a finitary Bloom algebra iff $a$ has a fixpoint - this is the same as in Example 3.2(b).

\section{Remark 8.4.}

(a) Recall from [10] the concept of an iterative algebra: it is an algebra $a: H A \rightarrow A$ such that every equation morphism $e: X \rightarrow H X+A$ with $X$ finitely presentable has a unique solution. That is, the algebra $[a, A]: H A+A \rightarrow A$ for $H(-)+A$ is finitary corecursive. Every iterative algebra is obviously finitary corecursive (for $H$ ). An example of a finitary corecursive algebra that is not iterative is the algebra of all binary trees with finitely many leaves, all of which are labelled in $Y$, see Example 4.7(3).

(b) Recall further from [10] that the category

$$
\text { Coalg }_{f} H
$$

of all coalgebras on finitely presentable objects of $\mathcal{A}$ is filtered, and the filtered colimit of the forgetful functor to $\mathcal{A}$,

$$
R=\operatorname{colim}\left\{X ;(X, e) \in \text { Coalg }_{f} H\right\}
$$

carries the structure of a coalgebra $i: R \rightarrow H R$. This structure is an isomorphism, and its inverse $\rho: H R \rightarrow R$ is the initial iterative algebra. Consequently, $R$ is a finitary Bloom algebra as well. Indeed:

Proposition 8.5. $R$ is the initial finitary corecursive algebra.

Proof. We know that $R$ is finitary corecursive because it is even iterative. Let $(A, a)$ be a finitary Bloom algebra. Given a (solution-preserving) homomorphism $h: R \rightarrow A$, for every $e: X \rightarrow H X$ in Coalg $_{f} H$ the triangle

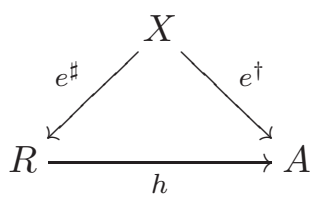

commutes, where $e^{\sharp}$ denotes the solution in $R$. As proved in [10], these morphisms $e^{\sharp}$ form the colimit cocone of $R$ (as a colimit of the forgetful functor of Coalg $H$ ). Thus, the above triangles determine, since $\dagger$ is functorial, a unique morphism $h$ which is solution-preserving. It remains to prove that $h$ is a homomorphism. For that recall from [10] that the algebra structure $\rho: H R \rightarrow R$ is defined as the inverse of the unique isomorphism $i: R \rightarrow H R$ with

$$
i \cdot e^{\sharp}=H e^{\sharp} \cdot e \text { for all } e: X \rightarrow H X \in \text { Coalg }_{f} H .
$$

Thus in order to prove $h \cdot \rho=a \cdot H h$ we use that $h=a \cdot H h \cdot i: R \rightarrow A$ which follows from the fact that $e^{\sharp}$ are collectively epic: $h \cdot e^{\sharp}=e^{\dagger}=a \cdot H e^{\dagger} \cdot e=a \cdot H h \cdot H e^{\sharp} \cdot e=a \cdot H h \cdot i \cdot e^{\sharp}$. 


\section{Example 8.6.}

(a) For $H X=X \times X+1$ the algebra $R$ consists of all binary trees that are rational, i. e., have finitely many subtrees up to isomorphism, see [25].

(b) More generally, given a finitary signature $\Sigma$ the polynomial functor $H_{\Sigma}$ : Set $\rightarrow$ Set with

$$
H_{\Sigma} X=\Sigma_{0}+\Sigma_{1} \times X+\Sigma_{2} \times X^{2}+\cdots
$$

has the initial iterative algebra

$$
R_{\Sigma}=\text { all rational } \Sigma \text {-trees. }
$$

(c) For the finite power-set functor $R$ is the algebra of all rational, finitely branching, strongly extensional trees in the sense of J. Worrell [34].

Theorem 8.7. Every object $Y$ generates a free finitary Bloom algebra. This is the coproduct $R \oplus F Y$ of $R$ and the free algebra $F Y$ in $\mathrm{Alg} H$.

Proof. The category Alg $H$ is locally finitely presentable, see [14, thus $\oplus$ exists in Alg $H$. The coproduct injection inl : $R \rightarrow R \oplus F Y$ induces a finitary Bloom algebra structure on $R \oplus F Y$, see Lemma 3.7. And we have a canonical morphism inr $\cdot \eta_{Y}: Y \rightarrow R \oplus F Y$ in $\mathcal{A}$.

The universal property of inr $\cdot \eta_{Y}$ is clear: let $(B, b, \ddagger)$ be a finitary Bloom algebra and $g: Y \rightarrow B$ a morphism in $\mathcal{A}$.

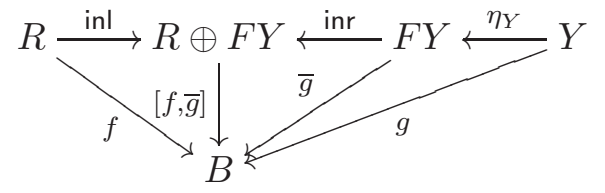

We have a unique homomorphism $\bar{g}: F Y \rightarrow B$ and, due to Proposition 8.5, a unique solution-preserving homomorphism $f: R \rightarrow B$. The unique homomorphism $[f, \bar{g}]: R \oplus$ $F Y \rightarrow B$ is solution-preserving since $f$ is and the Bloom algebra structure of $R \oplus F Y$ is induced by inl.

Corollary 8.8. $R \oplus F Y$ is a free finitary corecursive algebra on $Y$.

Indeed, the proof that solutions in $R \oplus F Y$ are unique is completely analogous to that of Theorem 4.6.

Example 8.9. Let $\Sigma$ be a finitary signature. The free finitary corecursive algebra $M^{*} Y=$ $R_{\Sigma} \oplus F_{\Sigma} Y$ is the algebra of all rational trees with finitely many leaves labelled in $Y$ and all other nodes (with $n$ successors) labelled by an $n$-ary operation in $\Sigma$ for $n=0,1,2, \ldots$

Notation 8.10. The monad of free finitary Bloom algebras is denoted by $\mathbb{M}_{H}^{*}$. It is defined on objects by assigning to $Y$ the underling object of $R \oplus F Y$. In the case of $H=H_{\Sigma}$ we write $\mathbb{M}_{\Sigma}^{*}$.

Theorem 8.11. The category of finitary Bloom algebras is isomorphic to the EilenbergMoore category of $\mathbb{M}_{H}^{*}$.

The proof is analogous to that of Theorem 4.15.

Remark 8.12. Analogous to the concept of a corecursive monad, see Definition 5.3, we call an ideal monad $\mathbb{S}$ finitary corecursive if every ideal equation morphism $e: X \rightarrow S X$ with $X$ finitely presentable has a unique solution $e^{\dagger}: X \rightarrow S Y$. The following result is completely analogous to Proposition 5.5 and Theorem 6.4 
Theorem 8.13. For every finitary endofunctor $H$ the monad $\mathbb{M}_{H}^{*}$ of free finitary corecursive algebras is a free finitary corecursive monad on $H$. This monad is ideal with the ideal HM and $\mu^{\prime}=H \mu$.

\section{Notation 8.14.}

(a) Let $\mathbb{F}$ be a full subcategory of $\mathcal{A}$ representing all finitely presentable objects. The functor category $\mathcal{A}^{\mathbb{F}}$ is equivalent to the category of all finitary endofunctors of $\mathcal{A}$.

(b) $\mathcal{A}_{\perp}^{\mathbb{F}}$ denotes the non-full subcategory of all strict finitary endofunctors (and strict natural transformations). The embedding $\mathcal{A}_{\perp}^{\mathbb{F}} \hookrightarrow \mathcal{A}^{\mathbb{F}}$ has a left adjoint $H \mapsto H_{\perp}$ where $H_{\perp} X=H X+1$.

(c) We denote by

$$
\mathcal{M}^{*}
$$

the monad on $\mathcal{A}^{\mathbb{F}}$ given by free finitary corecursive monads:

$$
\mathcal{M}^{*}: H \mapsto M_{H_{\perp}}^{*}
$$

More precisely $\mathcal{M}^{*}$ is the monad obtained by the composite adjoint situation

$$
\mathcal{A}^{\mathbb{F}} \stackrel{(-)_{\perp}}{\stackrel{\perp}{\leftrightarrows}} \mathcal{A}_{\perp}^{\mathbb{F}} \stackrel{\stackrel{M_{(-)}^{*}}{\stackrel{\perp}{\leftrightarrows}}}{\stackrel{U}{U}} \mathrm{FC}_{\perp}(\mathcal{A})
$$

where $U$ is the forgetful functor of the category $\mathrm{FC}_{\perp}(\mathcal{A})$ of all strict finitary corecursive monads.

Definition 8.15. A Bloom monad on $\mathcal{A}$ is an Eilenberg-Moore algebra for the monad $\mathcal{M}^{*}$.

Remark 8.16. Following Theorem 7.1, this concept is, for $\mathcal{A}=$ Set, completely analogous to iteration theories of Bloom and Ésik: whereas iteration theories formalizes equational properties of parametrized iteration, Bloom monads on Set formalize equational properties of non-parametrized iteration. But what are Bloom monads?

(1) Every Bloom monad is a finitary monad on $\mathcal{A}$. Indeed, let $\mathcal{F}$ be the free-monad on $\mathcal{A}^{\mathbb{F}}$ : to every finitary endofunctor $H$ it assigns the free monad $F_{H}$ on $H$. It is well-known that the Eilenberg-Moore algebras for $\mathcal{F}$ are precisely the finitary monads on $\mathcal{A}$, see [27].

For every $H$ in $\mathcal{A}^{\mathbb{F}}$ we have the unique monad morphism

$$
\phi_{H}: \mathcal{F}(H) \rightarrow \mathcal{M}^{*}(H)=M_{H_{\perp}}^{*}
$$

given by the universal property of $\mathcal{F}(H)$. These morphisms form components of a monad morphism $\phi: \mathcal{F} \rightarrow \mathcal{M}^{*}$ (over $\mathcal{A}^{\mathbb{F}}$ ). Thus, every Eilenberg-Moore algebra for $\mathcal{M}^{*}$ is automatically one for $\mathcal{F}$, too.

(2) Every Bloom monad $\mathbb{S}=(S, \eta, \mu)$ comes equipped with an operation $\dagger$ assigning to every equation morphism $e: X \rightarrow S X$ with $X$ finitely presentable and every object $Y$ a morphism $e^{\dagger}: X \rightarrow S Y$ which is a solution:

$$
e^{\dagger}=\mu_{Y} \cdot S e^{\dagger} \cdot e
$$

Indeed, the Eilenberg-Moore structure

$$
\sigma: M_{S_{\perp}}^{*} \rightarrow S
$$

is a monad morphism, and we have also the universal arrow (see Example 6.3)

$$
\bar{\kappa} \equiv S \stackrel{\text { inl }}{\longrightarrow} S_{\perp} \stackrel{\kappa}{\longrightarrow} M_{S_{\perp}}^{*}
$$


which, due to the unit law of $\sigma$, fulfils

$$
\sigma \cdot \bar{\kappa}=i d_{S} .
$$

For every equation morphism $e: X \rightarrow S X$ the unique strict solution (see Theorem 7.14) of $\bar{\kappa}_{X} \cdot e: X \rightarrow M_{S_{\perp}}^{*} X$ w.r.t. $\mathbb{M}_{S_{\perp}}^{*}$ is denoted by $e^{\ddagger}: X \rightarrow M_{S_{\perp}}^{*} Y$. Then

$$
e^{\dagger}=\sigma_{Y} \cdot e^{\ddagger}: X \rightarrow S Y
$$

is a (canonical) solution of $e$ w.r.t. $\mathbb{S}$. Indeed, the following diagram, where $\bar{\mu}$ denotes the multiplication of $\mathbb{M}_{S_{\perp}}^{*}$, commutes:

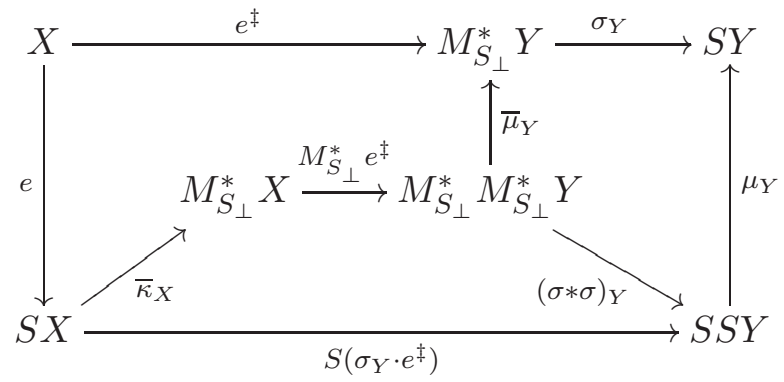

The left-hand part commutes since $e^{\ddagger}$ is a solution of $\bar{\kappa}_{X} \cdot e$. The right-hand part (with $\sigma * \sigma=S \sigma \cdot \sigma M_{S_{\perp}}^{*}$ ) commutes because $\sigma: \mathbb{M}_{S_{\perp}}^{*} \rightarrow \mathbb{S}$ is a monad morphism. And to prove the lower part, we just need to verify

$$
S e^{\ddagger}=\sigma_{M_{\perp}^{*} Y}^{*} \cdot M_{S_{\perp}}^{*} e^{\ddagger} \cdot \bar{\kappa}_{X}
$$

which follows easily from $\sigma_{X} \cdot \bar{\kappa}_{X}=i d$ and the naturality of $\sigma$.

Remark 8.17. (a) The operation $\dagger$ above satisfies all the equational laws that the formation of strict solutions in all finitary corecursive monads satisfies. This follows from the fact that the Bloom monad $(\mathbb{S}, \dagger)$ is by definition a quotient algebra (for $\mathcal{M}^{*}$ ) of the finitary corecursive monad $\left(\mathbb{M}_{S_{\perp}}^{*}, \ddagger\right)$.

(b) For the base category Set we can say more. Since $S$ is finitary, there exists a finitary signature $\Sigma$ such that $S$ is a quotient of $H_{\Sigma}$ (see [15]). Let $\Sigma_{\perp}=\Sigma \cup\{\perp\}$ be the extension by a nullary operation $\perp$. Then $S_{\perp}$ is a quotient of $H_{\Sigma_{\perp}}$. Indeed, there exists a signature $\Gamma$ such that for suitable natural transformations $\alpha_{1}, \alpha_{2}: H_{\Gamma} \rightarrow H_{\Sigma}$ we have a coequalizer

$$
H_{\Gamma} \stackrel{\alpha_{1}}{\underset{\alpha_{2}}{\longrightarrow}} H_{\Sigma_{\perp}} \stackrel{e}{\longrightarrow} S_{\perp},
$$

see [7]. The functor $\mathbb{M}_{(-)}^{*}$ of free finitary corecursive monads is a left adjoint, thus, it preserves coequalizers. Therefore $\mathcal{M} S_{\perp}$ is a quotient of $\mathcal{M} H_{\Sigma_{\perp}}$.

Consequently, a Bloom monad in Set is a finitary monad with a solution operation $\dagger$ satisfying all the equational laws that the operation of unique strict solutions for the rational-tree monads $\mathbb{M}_{\Sigma_{\perp}}^{*}$ satisfies.

In the following example we list some equational properties of $\dagger$ in Bloom monads. It is an open problem whether this list is complete in the sense that every equational property of $\dagger$ holding in all Bloom monads can be derived from the properties stated.

Example 8.18. Equational properties of $\dagger$ in Bloom monads. We use the terminology of the monograph [19]. 
(a) Fixpoint identity. This is the equation

$$
e^{\dagger}=\mu_{Y} \cdot S e^{\dagger} \cdot e
$$

of Definition 5.3 ,

(b) Functoriality (called functorial dagger implication in [19]). For every coalgebra homomorphism

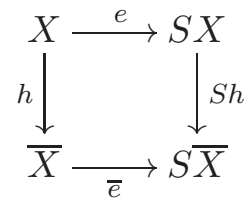

we have

$$
e^{\dagger}=\bar{e}^{\dagger} \cdot h
$$

To prove this recall that we can restrict ourselves to finitary corecursive monads where strict solutions are unique (Remark 8.17(a)). Thus, it is sufficient to observe that $\bar{e}^{\dagger} \cdot h$ solves $e$ :

$$
\mu_{Y} \cdot S\left(\bar{e}^{\dagger} \cdot h\right) \cdot e=\mu_{Y} \cdot S \bar{e}^{\dagger} \cdot \bar{e} \cdot h=\bar{e}^{\dagger} \cdot h
$$

and that it is strict. The latter follows from the fact that $\bar{e}^{\dagger}$ is strict for $\bar{e}$ and the subobjects $i_{n}: X_{n} \rightarrow X_{n-1}$ of Definition 7.11 for $e$ are related to the corresponding subobject $\bar{i}_{n}: \bar{X}_{n} \rightarrow \bar{X}_{n-1}$ for $\bar{e}$ by morphisms $h_{n}: X \rightarrow \bar{X}_{n}$ such that the squares below commute:

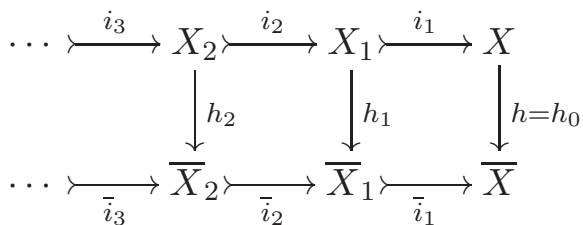

For example, $h_{1}$ is the unique morphism for which the following diagram commutes:

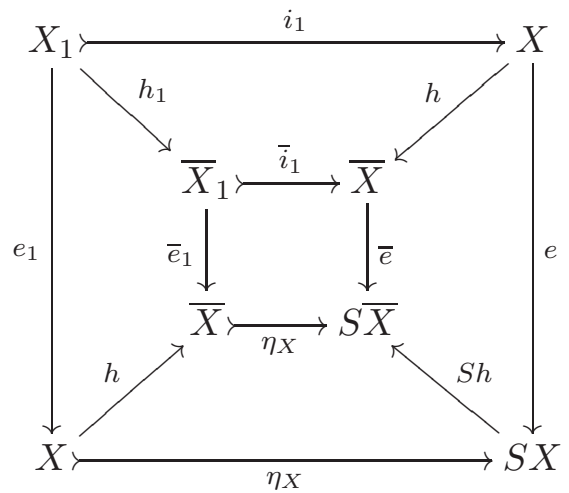

satisfying $h_{n-1} \cdot i_{n}=\bar{i}_{n} \cdot h_{n}$ (where $\left.h_{0}=h\right)$. Therefore, we obtain $h_{\infty}: X_{\infty} \rightarrow \bar{X}_{\infty}$ such that $h \cdot i_{\infty}=\bar{i}_{\infty} \cdot h_{\infty}$. This gives us the derived factorization

$$
\left(\bar{e}^{\dagger} \cdot h\right) \cdot i_{\infty}=\bar{e}^{\dagger} \cdot \bar{i}_{\infty} \cdot h_{\infty}=\perp \cdot ! \cdot i_{\infty}=\perp \cdot !
$$

(c) Parameter identity. Given $e: X \rightarrow S X$, with $X$ finitely presentable, and a morphism $h: Y \rightarrow S Z$, then the corresponding morphism of free Eilenberg-Moore algebras

$$
\hat{h}=\mu_{Z} \cdot S h: S Y \rightarrow S Z
$$


makes the triangle

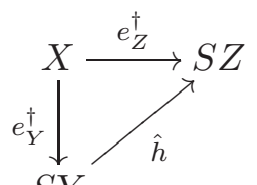

$S Y$

commutative. Indeed, $\hat{h} \cdot e_{Y}^{\dagger}$ is strict because $e_{Y}^{\dagger}$ is strict: from the strictness of $S$ we get $\hat{h} \cdot \perp=\perp$, thus

$$
\hat{h} \cdot e_{Y}^{\dagger} \cdot i_{\infty}=\hat{h} \cdot \perp \cdot !=\perp \cdot !
$$

And $\hat{h} \cdot e_{Y}^{\dagger}$ is a solution of $e$ in $S Z$ :

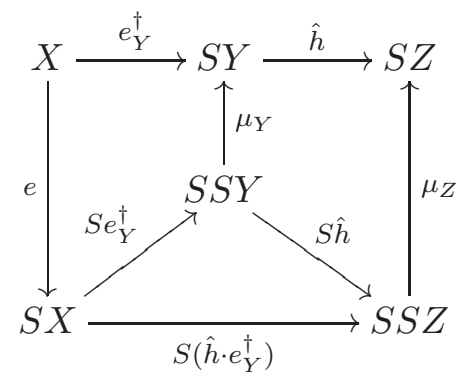

(d) Double iteration identity. For every $e: X \rightarrow S X$, form $\hat{e}: S X \rightarrow S X$, then we have

$$
e^{\dagger}=(\hat{e} \cdot e)^{\dagger}
$$

Indeed, $e^{\dagger}$ is a solution of $\hat{e} e=\mu_{X} \cdot S e \cdot e$ :

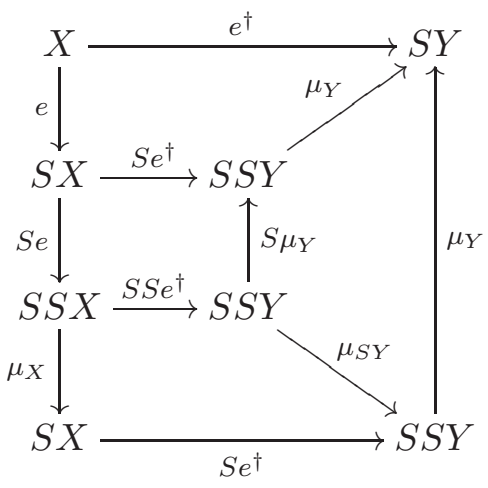

The upper part states $e^{\dagger}$ is a solution, and the middle part follows. The right-hand part is a monad axiom of $\mathbb{S}$ and the lower part is the naturality of $\mu$. The strictness of $e^{\dagger}$ w.r.t. $\hat{e} \cdot e$ is trivial: since $\mathbb{S}$ is an ideal monad on a hyper-extensive category, we 
have the following diagram of pullback squares:

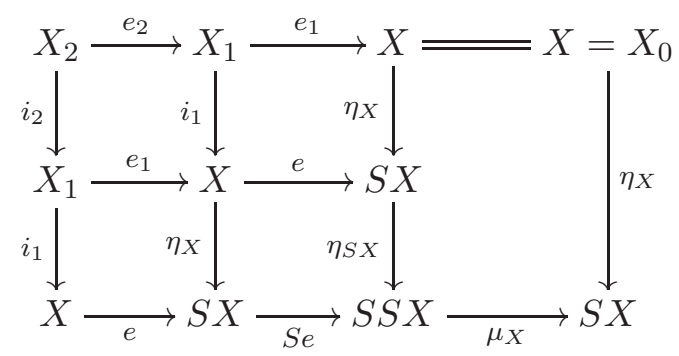

So the first derived subobject of $\hat{e} \cdot e$ is $X_{2}$, and similarly the $n$-th one is $X_{2 n}$, where the $X_{i}$ are the derived subobjects of $e$. If follows that $X_{\infty}$ is the same for $e$ and $\hat{e} \cdot e$, thus, $e^{\dagger}$ is a strict solution of $\hat{e} \cdot e$.

Observe that the above "double iteration" extends to " $n$ times iteration", e.g. for $n=3$ we get $e^{\dagger}=(\hat{e} \hat{e} e)^{\dagger}$.

(e) Dinaturality. Given morphisms $f: X \rightarrow S Z$ and $g: Z \rightarrow S X$ with $X$ and $Z$ finitely presentable, form equation morphisms

$$
\hat{g} \cdot f: X \rightarrow S X \quad \text { and } \quad \hat{f} \cdot g: Z \rightarrow S Z .
$$

Their solutions are related by the dinaturality equation

$$
(\hat{g} \cdot f)^{\dagger}=\widehat{(\hat{f} \cdot g)^{\dagger}} \cdot f: X \rightarrow S Y
$$

for every object $Y$.

Indeed, the right-hand side morphism is a solution of $\hat{g} \cdot f$ since it is the composite $\mu_{Y} \cdot S(\hat{f} \cdot g)^{\dagger} \cdot f$, and the following diagram

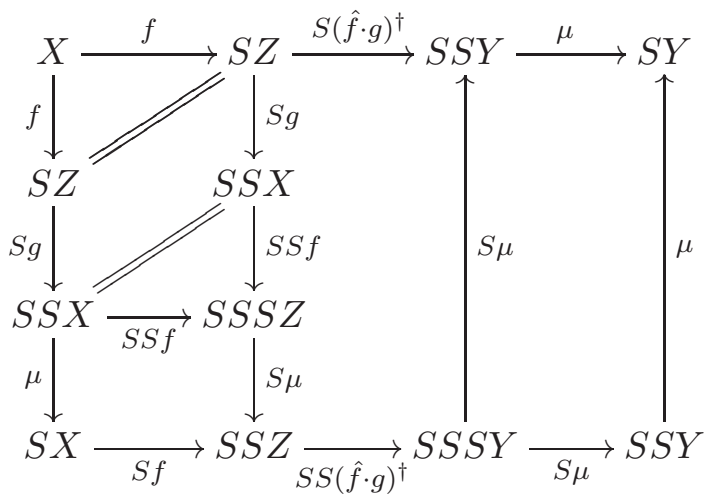

commutes: the middle part follows from $(\hat{f} \cdot g)^{\dagger}$ solving the equation morphism $\hat{f} \cdot g=$ $\mu_{Z} \cdot S f \cdot g$.

To see that $\widehat{(\hat{f} \cdot g)} \cdot f$ is a strict solution of $\hat{g} \cdot f$, we first need to relate the derived subobjects of $\hat{g} \cdot f$ and $f \cdot \hat{g}$. For this consider the two following chains of pullbacks:

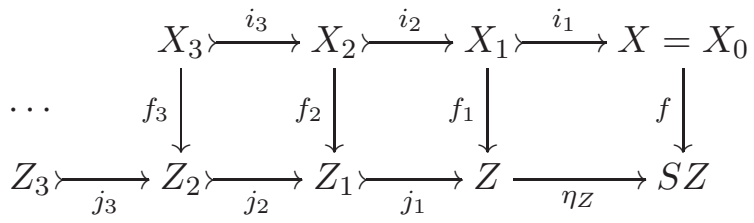




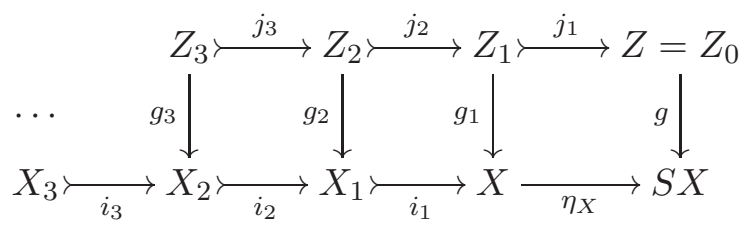

Using them and the fact that $\mathbb{S}$ is an ideal monad in connection with (hyper-)extensivity we can compute the derived subobjects of $\hat{g} \cdot f$ as follows (all squares in following the diagram are pullbacks):

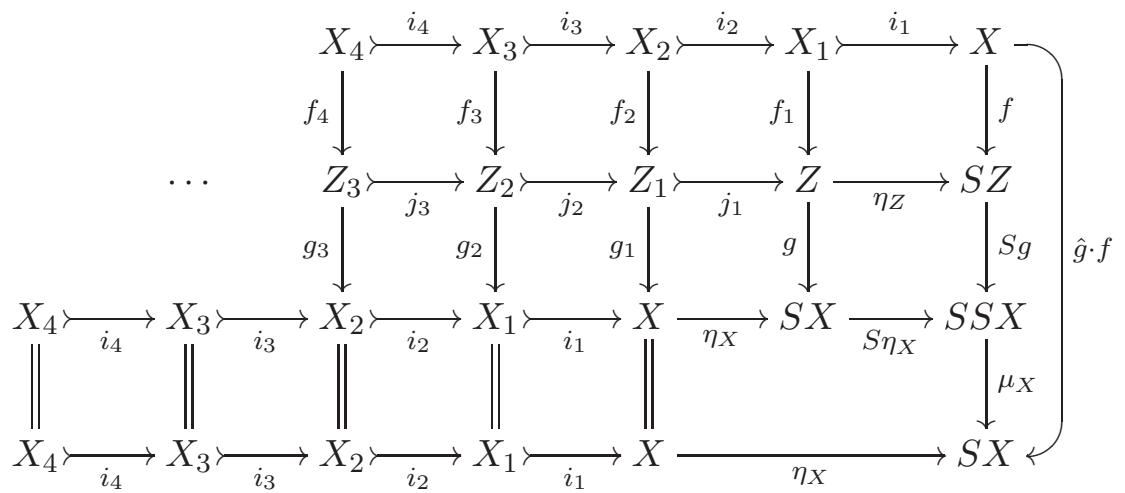

So we see that the first derived subobject of $\hat{g} \cdot f$ is $i_{1} \cdot i_{2}: X_{2} \longmapsto X$, and the second one is $i_{1} \cdot i_{2} \cdot i_{3} \cdot i_{4}: X_{4} \longmapsto X$ etc. similarly the $n$-th derived subobject of $\hat{f} \cdot g$ is

$$
j_{1} \cdot j_{2} \cdots j_{2 n}: Z_{2 n} \longmapsto Z \text {. }
$$

Now recall from [3, Lemma 6.4] that the intersections $X_{\infty}$ and $Z_{\infty}$ are obtained after finitely many steps in the computation of the derived subobjects, i. e. there exists an $n$ such that $X_{\infty}=X_{k}$ and $Z_{\infty}=Z_{k}$ for all $k \geq n$. Thus, we establish that $\overline{(\hat{f} \cdot g)^{\dagger} \cdot f}$ is a strict solution of $\hat{g} \cdot f$ by the commutative diagram below:

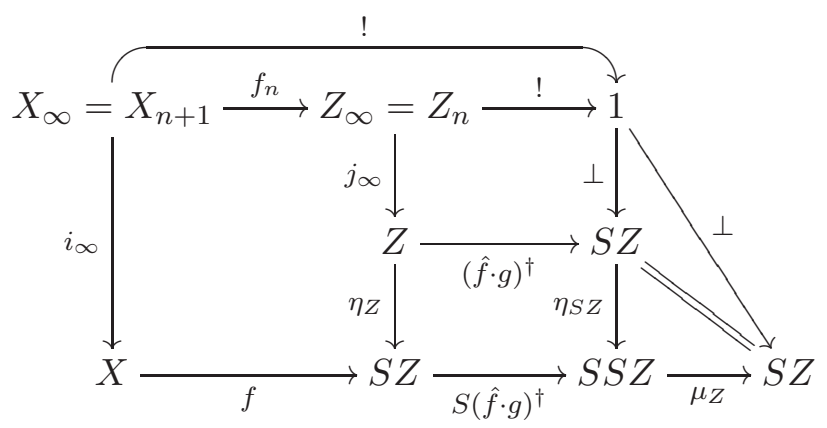

This completes the proof.

\section{Conclusions And Open Problems}

For coalgebras, recursivity caN be defined by the existence of unique algebra-to-coalgebra homomorphisms (no parameters are used). Or, equivalently, assuming the given endofunctor preserves weak pullbacks, by the unique solutions of all recursive systems with parameters. In contrast, in the dual situation we need to study non-equivalent variations. The present paper is dedicated to corecursive algebras $A$, where corecursivity means that every recursive 
system of equations represented by a coalgebra has a unique solution in $A$. The formulation above is strictly weaker than the concept of a completely iterative algebra, where every parametrized recursive system of equations has a unique solution. For example, if we consider the endofunctor $X \mapsto X \times X$ of one binary operation in Set, the algebra of all binary trees with finitely many leaves is corecursive, but not completely iterative.

The main result of our paper is the description of the free corecursive algebra on $Y$ as the coproduct $M Y=T \oplus F Y$ of the terminal coalgebra $T$ and the free algebra $F Y$ in the category of all algebras. The above example of binary trees is the free corecursive algebra $M 1$ on one generator. Our description is true for all accessible (= bounded) endofunctors on Set and, more generally, for all endofunctors on Set having free corecursive algebras. For accessible monos-preserving endofunctors on more general base categories (posets, groups, monoids etc.) the above description of the free corecursive algebras also holds.

We introduce the concept of a corecursive monad, a weakening of completely iterative monad. We prove that the assignment $Y \mapsto M Y=T \oplus F Y$ is the free corecursive monad on the given accessible endofunctor. And we characterize the Eilenberg-Moore algebras for this monad. We call them Bloom algebras in honor of Stephen Bloom. They play the analogous role that Elgot algebras, studied in [9], play for iterative monads: solutions of recursive equations are not required to be unique, but have to satisfy some "basic" properties. In the case of Bloom algebras, the only property needed is functoriality.

We further treat finitary equations: If we consider systems of recursive equations as coalgebras $e: X \rightarrow H X$, then finite systems of recursive equation are represented by coalgebras in which $X$ is a finite set (or more generally, a finitely presentable object). We can speak about finitary corecursive algebras as those in which these finite systems have unique solutions. We prove that if $R$ is the initial iterative algebra, then $R \oplus F Y$ is a free finitary corecursive algebra.

Another question is: what is the analogy of the notion of an iteration monad of S. Bloom and Z. Ésik [19] in the realm of corecursive algebras? We do not know the answer. But at least we can formulate the question precisely. The idea of iteration monads is to collect all "equational" properties that the operation $e \mapsto e^{\dagger}$ of solving recursive systems $e$ has in trees for a signature. This can be understood as forming the monad of free iterative theories (or monads) on the category Set $^{\mathbb{F}}$ of sets in context, and characterizing monadic algebras: these are, as proved in [12, precisely the iteration theories of S. Bloom and Z. Ésik that are functorial. So the open problem we state is this: form the monad of free finitary corecursive theories on Set $^{\mathbb{F}}$, what are its monadic algebras? We called them Bloom monads, and listed some of their properties.

\section{REFERENCES}

[1] Peter Aczel, Jiří Adámek, Stefan Milius, and Jiří Velebil. Infinite trees and completely iterative theories: A coalgebraic view. Theoret. Comput. Sci., 300:1-45, 2003.

[2] Jiří Adámek. Free algebras and automata realizations in the language of categories. Comment. Math. Univ. Carolince, 15:589-602, 1974.

[3] Jiří Adámek, Reinhard Börger, Stefan Milius, and Jiří Velebil. Iterative algebras: How iterative are they? Theory Appl. Categ., 19:61-92, 2008.

[4] Jiří Adámek, Mahdieh Haddadi, and Stefan Milius. From corecursive algebras to corecursive monads. In Proc. 4th Conference on Algebra and Coalgebra in Computer Science (CALCO'11), volume 6859 of Lecture Notes Comput. Sci., pages 55-69. Springer, 2011. 
[5] Jiří Adámek, Horst Herrlich, and George E. Strecker. Abstract and concrete categories. Dover Publications, 2009.

[6] Jiří Adámek and Václav Koubek. On the greatest fixed point of a set functor. Theoretical Computer Science, 150:57-75, 1995.

[7] Jiří Adámek, Stefan Milius, and Lawrence S. Moss. On finitary functors and their presentation. In Dirk Pattinson and Lutz Schröder, editors, Proc. Coalgebraic Methods in Computer Science (CMCS'12), volume 7399 of Lecture Notes Comput. Sci. Springer, 2012.

[8] Jiří Adámek, Stefan Milius, and Jiří Velebil. On coalgebras based on classes. Theoret. Comput. Sci., 316:3-23, 2004.

[9] Jiří Adámek, Stefan Milius, and Jiří Velebil. Elgot algebras. Log. Methods Comput. Sci., 2(5:4):31 pp., 2006.

[10] Jiří Adámek, Stefan Milius, and Jiří Velebil. Iterative algebras at work. Math. Structures Comput. Sci., 16(6):1085-1131, 2006.

[11] Jiří Adámek, Stefan Milius, and Jiří Velebil. What are iteration theories? In L. Kučera and A. Kučera, editors, Proc. of the 32nd International Symposium on Mathematical Foundations of Computer Science (MFCS), volume 4708 of Lecture Notes Comput. Sci., pages 240-252. Springer, 2007.

[12] Jiří Adámek, Stefan Milius, and Jiří Velebil. Elgot theories: a new perspective of the equational properties of iteration. Math. Structures Comput. Sci., 21(2):417-480, 2011.

[13] Jiří Adámek and Hans-Eberhard Porst. On tree coalgebras and coalgebra presentations. Theoret. Comput. Sci., 311:257-283, 2004.

[14] Jiří Adámek and Jiří Rosický. Locally presentable and accessible categories. Cambridge University Press, 1994.

[15] Jiří Adámek and Věra Trnková. Automata and Algebras in Categories. Kluwer Academic Publishers, 1990.

[16] Jiří Adámek and Věra Trnková. Relatively terminal coalgebras. J. Pure Appl. Algebra, 216(8-9):18871895, August 2012.

[17] Pierre America and Jan Rutten. Solving reflexive domain equations in a category of complete metric. J. Comput. System Sci., 39:343-375, 1989.

[18] Michael Barr. Coequalizers and free triples. Math. Z., 116:307-322, 1970.

[19] Stephen L. Bloom and Zoltán Ésik. Iteration Theories: the equational logic of iterative processes. EATCS Monographs on Theoretical Computer Science. Springer, 1993.

[20] Francis Borceux. Handbook of categorical algebra 2. Cambridge Univ. Press. Prentice Hall, 1994.

[21] Venanzio Capretta, Tarmo Uustalu, and Varmo Vene. Corecursive algebras: A study of general structured corecursion. Lecture Notes Comput. Sci. 5902, pages 84-100, 2009.

[22] Calvin C. Elgot. Monadic computation and iterative algebraic theories. In H. E. Rose and J. C. Sheperdson, editors, Logic Colloquium '73, Amsterdam, 1975. North-Holland Publishers.

[23] Calvin C. Elgot, Stephen L. Bloom, and Ralph Tindell. On the algebraic structure of rooted trees. Comput. System Sci, 16:361-399, 1978.

[24] Neil Ghani and Tarmo Uustalu. Coproducts of ideal monads. Theor. Inform. Appl., 38(4):321-342, 2004.

[25] Susanna Ginali. Regular trees and the free iterative theory. J. Comput. System Sci., 18:228-242, 1979.

[26] G. Max Kelly and A. John Power. Adjunctions whose counits are coequalizers, and presentations of finitary enriched monads. J. Pure Appl. Algebra, 89:163-179, 1993.

[27] Stephen Lack. On the monadicity of finitary monads. J. Pure Appl. Algebra, 140:65-73, 1999.

[28] Stefan Milius. Completely iterative algebras and completely iterative monads. Inform. and Comput., 196:1-41, 2005.

[29] Gerhard Osius. Categorical set theory: a characterization of the category of set. J. Pure Appl. Algebra, 4:79-119, 1974.

[30] Alex Simpson and Gordon D. Plotkin. Complete axioms for categorical fixed-point operators. In Proc. 15th Symposium on Logic in Computer Science (LICS'00), pages 30-41. IEEE Computer Society, 2000.

[31] Paul Taylor. Towards a unified treatment of induction 1: the general recursion theorem. Preprint 1995-6, available at http://www.paultaylor .eu/ordinals\#towuti.

[32] Paul Taylor. Practical Foundations of Mathematics. Cambridge University Press, 1999.

[33] Věra Trnková, Jiří Adámek, Václav Koubek, and Jan Reiterman. Free algebras, input processes and free monads. Comment. Math. Univ. Carolinœe, 16:339-351, 1975. 
[34] James Worrell. On the final sequence of a finitary set functor. Theoret. Comput. Sci., 338:184-199, 2005.

This work is licensed under the Creative Commons Attribution-NoDerivs License. To view a copy of this license, visit http://creativecommons.org/licenses/by-nd/2.0/ or send a letter to Creative Commons, 171 Second St, Suite 300, San Francisco, CA 94105, USA, or Eisenacher Strasse 2, 10777 Berlin, Germany 